\title{
ARITHMETIC OF COMMUTATIVE SEMIGROUPS WITH A FOCUS ON SEMIGROUPS OF IDEALS AND MODULES
}

\author{
YUSHUANG FAN, ALFRED GEROLDINGER, FLORIAN KAINRATH, AND SALVATORE TRINGALI
}

\begin{abstract}
Let $H$ be a commutative semigroup with unit element such that every non-unit can be written as a finite product of irreducible elements (atoms). For every $k \in \mathbb{N}$, let $\mathscr{U}_{k}(H)$ denote the set of all $\ell \in \mathbb{N}$ with the property that there are atoms $u_{1}, \ldots, u_{k}, v_{1}, \ldots, v_{\ell}$ such that $u_{1} \cdot \ldots \cdot u_{k}=v_{1} \cdot \ldots \cdot v_{\ell}$ (thus, $\mathscr{U}_{k}(H)$ is the union of all sets of lengths containing $k$ ).

The Structure Theorem for Unions states that, for all sufficiently large $k$, the sets $\mathscr{U}_{k}(H)$ are almost arithmetical progressions with the same difference and global bound. We present a new approach to this result in the framework of arithmetic combinatorics, by deriving, for suitably defined families of subsets of the non-negative integers, a characterization of when the Structure Theorem holds.

This abstract approach allows us to verify, for the first time, the Structure Theorem for a variety of possibly non-cancellative semigroups, including semigroups of (not necessarily invertible) ideals and semigroups of modules. Furthermore, we provide the very first example of a semigroup (actually, a locally tame Krull monoid) that does not satisfy the Structure Theorem.
\end{abstract}

\section{INTRODUCTION}

The focus of factorization theory has been so far on the arithmetic of noetherian domains, Krull domains, semigroups of invertible ideals, and semigroups of modules, where the involved semigroups (of ring elements, ideals, or modules), both in the commutative and in the non-commutative setting, are cancellative. The best investigated means for describing the arithmetic of non-factorial semigroups are sets of lengths and invariants derived from them, such as sets of distances, elasticities, and unions of sets of lengths. To fix notation, we recall some definitions that lie at the center of our interest.

Let $H$ be a commutative semigroup and let $k$ be a positive integer. Then we use $\mathscr{U}_{k}(H)$ for the set of all $\ell \in \mathbb{N}$ with the property that there are atoms $u_{1}, \ldots, u_{k}, v_{1}, \ldots, v_{\ell} \in H$ such that $u_{1} \cdot \ldots \cdot u_{k}=v_{1} \cdot \ldots \cdot v_{\ell}$. Moreover, $\rho_{k}(H)=\sup \mathscr{U}_{k}(H) \in \mathbb{N}_{0} \cup\{\infty\}$ denotes the $k$-th elasticity of $H$.

Unions of sets of lengths were first studied by Chapman and Smith in the setting of Dedekind domains $R$ with finite class group $([8,9])$. Their focus was on the invariants $\left|\mathscr{U}_{k}(R)\right|$ and $\rho_{k}(R)$. The Structure Theorem for Unions states that, for all sufficiently large $k$, the sets $\mathscr{U}_{k}(H)$ are almost arithmetical progressions with the same difference and global bound. It was first proved for commutative cancellative semigroups with finite set of distances satisfying a natural growth condition for the $\rho_{k}(H)$ ([19, Theorem 4.2]), and later generalized to possibly non-commutative cancellative semigroups ([20, Theorem 2.6]). These abstract results served as crucial tools for studying unions of sets of lengths in settings ranging

2010 Mathematics Subject Classification. 11P70, 13A05, 13F05, 16D70, 20M12, $20 \mathrm{M} 13$.

Key words and phrases. Factorizations, sets of lengths, direct-sum decomposition, Krull monoids.

This work was supported by the Austrian Science Fund FWF, Project Number M 1900-N39, the National Natural Science Foundation of China (NSFC), Project No. 11401542, and the China Scholarship Council (CSC). 
from numerical monoids to Mori domains, including semigroups of modules and maximal orders in central simple algebras (see, for example, $[2,4,39,26])$. In special cases the unions $\mathscr{U}_{k}(H)$ are not only almost arithmetical progressions (as predicted by the Structure Theorem), but even arithmetical progressions. This holds true, among others, for Krull monoids with the property that every class contains a prime divisor ([21, Theorem 3.1.3]). In this case the study of the elasticities $\rho_{k}(H)$ has received much attention in recent literature $([22,17])$.

The goal of the present paper is twofold. First, we want to gain a better understanding of when the Structure Theorem for Unions holds true, and second, we want to do this in a setting that allows us to handle certain semigroups of ideals and semigroups of modules which are not necessarily cancellative.

In Section 2, we study some suitably defined families of sets of non-negative integers (systems of sets of lengths of semigroups are such families), and for these families we derive a characterization of when the Structure Theorem for Unions holds true (Theorem 2.2). In Section 3, we first consider abstract monoids, defined as commutative associative semigroups with a unit element for which an equation of the form $a u=a$ implies that $u$ is invertible. Our main results in this setting are Theorems 3.5 and 3.6. In Subsections 3.2 and 3.3, we apply the above results to semigroups of ideals in one-dimensional noetherian domains (Corollary 3.8) and to semigroups of modules of finite representation type (Corollary 3.9). In Section 4, we provide the very first example of a semigroup whose system of sets of lengths does not satisfy the Structure Theorem for Unions. Our example is, actually, a cancellative locally tame Krull monoid with finite set of distances (Theorem 4.2).

\section{Directed families of SUbSETS OF $\mathbb{N}_{0}$}

For $a, b \in \mathbb{R} \cup\{ \pm \infty\}$ we let $\llbracket a, b \rrbracket=\{x \in \mathbb{Z}: a \leq x \leq b\}$ be the discrete interval between $a$ and $b$. Given a set $X$, we write $\mathscr{P}(X)$ for the power set of $X$. We denote by $\mathbb{N}$ the set of positive integers and take $\mathbb{N}_{0}=\mathbb{N} \cup\{0\}$. By convention, we assume inf $\varnothing=\infty$ and $\sup \varnothing=0$.

Let $G$ be an additive abelian group, $n \in \mathbb{N}$, and $A, B \subseteq G$ subsets. Then $A+B=\{a+b: a \in A, b \in B\}$ is the sumset of $A$ and $B, n A=A+\ldots+A$ the $n$-fold sumset of $A$, and $n \cdot A=\{n a: a \in A\}$ the dilation of $A$ by $n$.

Let $L \subseteq \mathbb{N}_{0}$ be a subset. We denote by $L^{+}=L \cap \mathbb{N}$ the set of positive elements of $L$. The elasticity $\rho(L)$ of $L$ is then defined as $\rho(L)=\sup L^{+} / \min L^{+}$if $L^{+} \neq \varnothing$ and $\rho(L)=1$ if $L^{+}=\varnothing$. A positive integer $d \in \mathbb{N}$ is called a distance of $L$ if there are $k, \ell \in L$ with $\ell-k=d$ and the interval $\llbracket k, \ell \rrbracket$ contains no further elements of $L$. We let $\Delta(L)$ denote the set of distances of $L$. Thus $L$ is an arithmetical progression (AP for short) if $L \neq \varnothing$ and $|\Delta(L)| \leq 1$. Given $d \in \mathbb{N}$ and $M \in \mathbb{N}_{0}$, we call $L$ an almost arithmetical progression (AAP for short) with difference $d$ and bound $M$ if

- $L \subseteq y+d \cdot \mathbb{Z}$ for some $y \in \mathbb{Z}$, and

- $L \cap \llbracket \inf L+M, \sup L-M \rrbracket$ is an AP with difference $d$.

Clearly, every AAP with difference $d$ and bound $M=0$ is an AP with difference $d$, and conversely. By definition, AAPs are non-empty and they may be either finite or infinite.

Let $\mathscr{L}$ be a family of subsets of $\mathbb{N}_{0}$. For each $k \in \mathbb{N}_{0}$, we define

$$
\mathscr{L}_{k}=\{L \in \mathscr{L}: k \in L\} \quad \text { and } \quad \mathscr{U}_{k}(\mathscr{L})=\bigcup_{L \in \mathscr{L}_{k}} L,
$$


and we set $\lambda_{k}(\mathscr{L})=\inf \mathscr{U}_{k}(\mathscr{L})$ and $\rho_{k}(\mathscr{L})=\sup \mathscr{U}_{k}(\mathscr{L})$. We call

- $\rho(\mathscr{L})=\sup \{\rho(L): L \in \mathscr{L}\}$ the elasticity of $\mathscr{L}$,

- $\Delta(\mathscr{L})=\bigcup_{L \in \mathscr{L}} \Delta(L)$ the set of distances of $\mathscr{L}$, and

- the elements of $\Delta(\mathscr{L})$ the distances of $\mathscr{L}$.

We say that the family $\mathscr{L}$

(i) satisfies the Structure Theorem for Unions if there are $d \in \mathbb{N}$ and $M \in \mathbb{N}_{0}$ such that $\mathscr{U}_{k}(\mathscr{L})$ is an AAP with difference $d$ and bound $M$ for all sufficiently large $k \in \mathbb{N}$;

(ii) is directed if $1 \in L$ for some $L \in \mathscr{L}$ and, for all $L_{1}, L_{2} \in \mathscr{L}$, there is $L^{\prime} \in \mathscr{L}$ with $L_{1}+L_{2} \subseteq L^{\prime}$.

In this section we study directed families of subsets of $\mathbb{N}_{0}$ and characterize when they satisfy the Structure Theorem for Unions (Theorem 2.2). Such families play a prominent role in factorization theory, and here is the main example we have in mind.

Example 2.1. Let $H$ be a multiplicative semigroup, $H^{\times}$the group of invertible elements of $H$, and $A \subseteq H \backslash H^{\times}$a non-empty subset such that every $a \in H \backslash H^{\times}$can be written as a finite product of elements from $A$. If $a \in H \backslash H^{\times}$, then

$$
\mathrm{L}(a)=\left\{k \in \mathbb{N}: a=u_{1} \cdot \ldots \cdot u_{k} \text { with } u_{1}, \ldots, u_{k} \in A\right\} \subseteq \mathbb{N}
$$

is the set of lengths of $a$ (relative to $A$ ). For convenience we set $\mathrm{L}(a)=\{0\}$ for all $a \in H^{\times}$. Then $\mathscr{L}(H)=\{\mathrm{L}(a): a \in H\}$ is the system of sets of lengths of $H$ (relative to $A$ ). If $a \in A$, then $1 \in \mathrm{L}(a)$, and if $a_{1}, a_{2} \in H$, then $\mathrm{L}\left(a_{1}\right)+\mathrm{L}\left(a_{2}\right) \subseteq \mathrm{L}\left(a_{1} a_{2}\right)$. Thus $\mathscr{L}(H)$ is a directed family of subsets of $\mathbb{N}_{0}$.

The most natural choice of a generating set $A$ is the set of atoms of the semigroup and we study this situation in detail in Section 3. Also other generating sets have been, however, studied in the literature (e.g., [7]), and the main results of this section apply to them too. Clearly, every family $\mathscr{L}$ of subsets of $\mathbb{N}_{0}$, which is closed under set addition and contains some $L$ with $1 \in L$, is directed (see Subsection 3.4). Yet, systems of sets of lengths are not, in general, closed under set addition ([29]).

With the above in mind, we present the main result of this section.

Theorem 2.2. Let $\mathscr{L}$ be a directed family of subsets of $\mathbb{N}_{0}$ such that $\Delta(\mathscr{L})$ is finite non-empty and set $\delta=\min \Delta(\mathscr{L})$.

(1) Let $l \in \mathbb{N}_{0}$ such that $\{l, l+\delta\} \subseteq L$ for some $L \in \mathscr{L}$. Then $q=\frac{1}{\delta} \max \Delta(\mathscr{L})$ is a non-negative integer and the following statements are equivalent:

(a) $\mathscr{L}$ satisfies the Structure Theorem for Unions.

(b) There exists $M \in \mathbb{N}_{0}$ such that $\mathscr{U}_{k}(\mathscr{L}) \cap \llbracket \rho_{k-l q}(\mathscr{L})+l q, \rho_{k}(\mathscr{L})-M \rrbracket$ is either empty or an AP with difference $\delta$ for all sufficiently large $k$.

(2) Assume that $\rho(\mathscr{L})<\infty$ and there exists $L^{*} \in \mathscr{L}$ such that $\rho\left(L^{*}\right)=\rho(\mathscr{L})$ and $L^{*}$ is an $A P$ with difference $\delta$. Then $\mathscr{U}_{k}(\mathscr{L})$ is an AP with difference $\delta$ for all sufficiently large $k$.

Some comments about Condition $(1)(\mathrm{b})$ of Theorem 2.2 are in order. Suppose first that $\rho_{k_{0}}(\mathscr{L})=\infty$ for some $k_{0} \in \mathbb{N}$. Then it is found that $\rho_{k}(\mathscr{L})=\infty$ for all $k \geq k_{0}$ (see Lemma $2.4(3)$ ), and hence Condition $(1)(b)$ in the theorem holds, because $\llbracket \rho_{k-l q}(\mathscr{L})+l q, \rho_{k}(\mathscr{L})-M \rrbracket$ is empty. Assume, on the other hand, that $\rho_{k}(\mathscr{L})<\infty$ and $\rho_{k+1}(\mathscr{L})-\rho_{k}(\mathscr{L}) \leq K$ for all $k \in \mathbb{N}$, where $K \in \mathbb{N}$ does not depend on $k$. Then, 
in the notation of Theorem 2.2, we have

$$
\rho_{k}(\mathscr{L})-\rho_{k-l q}(\mathscr{L})=\sum_{i=k-l q}^{k-1}\left(\rho_{i+1}(\mathscr{L})-\rho_{i}(\mathscr{L})\right) \leq l q K,
$$

with the result that Condition (1)(b) is still verified (now with $M=l q K+1$ ).

Corollary 2.3. Let $\mathscr{L}$ be a directed family of subsets of $\mathbb{N}_{0}$ such that $\Delta(\mathscr{L})$ is finite non-empty and the Structure Theorem for Unions holds.

(1) If $\rho_{k}(\mathscr{L})<\infty$ for all $k \in \mathbb{N}$, then there is an $M \in \mathbb{N}_{0}$ such that $\mathscr{U}_{k}(\mathscr{L})$ is an AAP with difference $\min \Delta(\mathscr{L})$ and bound $M$ for every $k \in \mathbb{N}$.

(2) We have that

$$
\lim _{k \rightarrow \infty} \frac{\left|\mathscr{U}_{k}(\mathscr{L})\right|}{k}=\sup _{k \geq 1} \frac{\left|\mathscr{U}_{k}(\mathscr{L})\right|-1}{k}=\frac{1}{\min \Delta(\mathscr{L})}\left(\rho(\mathscr{L})-\frac{1}{\rho(\mathscr{L})}\right) .
$$

As mentioned in the Introduction, first results enforcing the validity of the Structure Theorem for Unions (together with the statements of Corollary 2.3) were established in [19, Theorem 4.2] and [20, Theorem 2.6]. The formula (2.1) was first derived in [8], for Dedekind domains with finite class group where each class contains a prime ideal. Note that, in this case, $\min \Delta(R)=1$ and $\rho(R)=\frac{1}{2} \mathrm{D}(G)$; hence the formula in [8] coincides with (2.1). A result along the lines of point (2) of Theorem 2.2 was first established in [18].

In order to prove Theorem 2.2, we proceed in a series of lemmas. For the rest of this section, $\mathscr{L}$ will be a directed family of subsets of $\mathbb{N}_{0}$. To ease notation, we set

$$
\rho=\rho(\mathscr{L}) \text { and } \Delta=\Delta(\mathscr{L}),
$$

and for every $k \in \mathbb{N}_{0}$ we take

$$
\mathscr{U}_{k}=\mathscr{U}_{k}(\mathscr{L}), \quad \rho_{k}=\rho_{k}(\mathscr{L}), \quad \text { and } \quad \lambda_{k}=\lambda_{k}(\mathscr{L}) .
$$

If $n \in \mathbb{N}$ and $L_{1}, \ldots, L_{n} \in \mathscr{L}$, then for all $q_{1}, \ldots, q_{n} \in \mathbb{N}$ there is $L \in \mathscr{L}$ for which $q_{1} L_{1}+\ldots+q_{n} L_{n} \subseteq L$. In particular, for every $k \in \mathbb{N}$ there exists $L \in \mathscr{L}$ with $k \in L$, whence $\mathscr{L}_{k}$ and $\mathscr{U}_{k}$ are non-empty.

Lemma 2.4. Let $h, k \in \mathbb{N}_{0}$. The following conditions hold:

(1) $h \in \mathscr{U}_{k}$ if and only if $k \in \mathscr{U}_{h}$.

(2) $\mathscr{U}_{h}+\mathscr{U}_{k} \subseteq \mathscr{U}_{h+k}$.

(3) If $\mathscr{U}_{h}$ and $\mathscr{U}_{k}$ are non-empty, then $\lambda_{h+k} \leq \lambda_{h}+\lambda_{k} \leq h+k \leq \rho_{h}+\rho_{k} \leq \rho_{h+k}$.

Proof. (1) Let $h \in \mathscr{U}_{k}$. There then exists $L \in \mathscr{L}_{k}$ such that $h \in L$, and hence $L \in \mathscr{L}_{h}$. It follows that $k \in \mathscr{U}_{h}$, since $k \in L \subseteq \mathscr{U}_{h}$, and this is enough to conclude (by symmetry).

(2) The claim is obvious if $\mathscr{U}_{h}=\mathscr{U}_{0}=\varnothing$ or $\mathscr{U}_{k}=\mathscr{U}_{0}=\varnothing$. Otherwise, pick $x \in \mathscr{U}_{h}$ and $y \in \mathscr{U}_{k}$. Then $x \in L_{h}$ and $y \in L_{k}$ for some $L_{h} \in \mathscr{L}_{h}$ and $L_{k} \in \mathscr{L}_{k}$, and since $\mathscr{L}$ is directed, there exist $L^{\prime} \in \mathscr{L}$ such that $x+y \in L_{h}+L_{k} \subseteq L^{\prime}$. Then $L^{\prime} \in \mathscr{L}_{h+k}$, and hence $x+y \in \mathscr{U}_{h+k}$, because $h+k \in L_{h}+L_{k}$. This implies that $\mathscr{U}_{h}+\mathscr{U}_{k} \subseteq \mathscr{U}_{h+k}$.

(3) It is sufficient to consider that, for all non-empty sets $X, Y \subseteq \mathbb{R}$, we have $\inf (X+Y)=\inf X+\inf Y$ and $\sup (X+Y)=\sup X+\sup Y$, and in addition $\inf Y \leq \inf X \leq \sup X \leq \sup Y$ if $X \subseteq Y$.

Lemma 2.5. Let $k \in \mathbb{N}$. 
(1) $\rho_{k}=\sup \{\sup L: L \in \mathscr{L}, \inf L \leq k\}=\sup \left\{\sup L: L \in \mathscr{L}_{k}\right\}$.

(2) If $\rho_{k}<\infty$ and $\rho_{k}=\sup L$ for some $L \in \mathscr{L}$ with $\inf L \leq k$, then $\inf L=k$ and $L \in \mathscr{L}_{k}$.

(3) $\rho_{k} \geq \sup \{\sup L: L \in \mathscr{L}, \inf L=k\}$, and equality holds if $\rho_{k}<\infty$.

Proof. For ease of exposition, set $\rho_{k}^{\prime}=\sup \{\sup L: L \in \mathscr{L}, \inf L \leq k\}, \rho_{k}^{\prime \prime}=\sup \left\{\sup L: L \in \mathscr{L}_{k}\right\}$, and $\bar{\rho}_{k}=\sup \{\sup L: L \in \mathscr{L}, \inf L=k\}$. Then, pick $L_{*} \in \mathscr{L}$ such that $1 \in L_{*}$.

(1) We have to show that $\rho_{k}=\rho_{k}^{\prime}=\rho_{k}^{\prime \prime}$. To begin, suppose for a contradiction that $\rho_{k}^{\prime \prime}<\rho_{k}$. There then exists $h \in \mathscr{U}_{k}$ such that $\rho_{k}^{\prime \prime}<h \leq \rho_{k}$. But $\mathscr{U}_{k}=\bigcup_{L \in \mathscr{L}_{k}} L$ yields that $h \in L$ for some $L \in \mathscr{L}_{k}$, with the result that $\sup L \leq \rho_{k}^{\prime \prime}<h \leq \sup L$, which is impossible.

It follows that $\rho_{k} \leq \rho_{k}^{\prime \prime}$, and it is clear from the definitions that $\rho_{k}^{\prime \prime} \leq \rho_{k}^{\prime}$, so we are left to show that $\rho_{k}^{\prime} \leq \rho_{k}$. For this, assume to the contrary that $\rho_{k}<\rho_{k}^{\prime}$. Then there is a set $L \in \mathscr{L}$ for which

$$
\inf L \leq k \quad \text { and } \quad \rho_{k}<\sup L \leq \rho_{k}^{\prime} .
$$

In particular, there exists $l \in L$ with $l \leq k$, and $\mathscr{L}$ being directed implies that $k=l+(k-l) \in$ $L+(k-l) L_{*} \subseteq \bar{L}$ for some $\bar{L} \in \mathscr{L}$. Thus $\bar{L} \in \mathscr{L}_{k}$, viz. $\bar{L} \subseteq \mathscr{U}_{k}$, and we have $\sup L \leq \sup \bar{L} \leq \rho_{k}$, which contradicts (2.2) and concludes the proof of point (1) of the lemma.

(2) Let $\rho_{k}=\sup L<\infty$ for some $L \in \mathscr{L}$ with $\inf L \leq k$. Accordingly, let $l \in L$ such that $l \leq k$. As before, we have (by the directedness of $\mathscr{L}$ ) that $k \in L+(k-l) L_{*} \subseteq \bar{L}$ for some $\bar{L} \in \mathscr{L}$. This implies $\rho_{k} \geq \sup \bar{L} \geq \sup L+k-l \geq \rho_{k}$, which is possible only if $l=k$. But $l$ is an arbitrary integer in $L$ that is $\leq k$, so we can conclude that $\inf L=k$, and hence $L \in \mathscr{L}_{k}$.

(3) It is clear from point (1) that $\bar{\rho}_{k} \leq \rho_{k}^{\prime}=\rho_{k}$, and the rest is straightforward by point (2).

The next propositions provide an interesting connection among the parameters we have so far introduced. In the proof of the first of them, we will make use of the following result, often referred to as Fekete's Lemma (see [36, Pt. I, Chap. 3, Problem No. 98, pp. 23 and 198]).

Lemma 2.6. Let $\left(x_{k}\right)_{k \geq 1}$ be a sequence with values in $\mathbb{R} \cup\{\infty\}$ such that $x_{h}+x_{k} \leq x_{h+k}$ for all $h, k \in \mathbb{N}$. Then the limit of $\frac{1}{k} x_{k}$ as $k \rightarrow \infty$ exists and is equal to $\sup _{k \geq 1} \frac{1}{k} x_{k}$.

Proposition 2.7. We have

$$
\rho=\sup _{k \geq 1} \frac{\rho_{k}}{k}=\lim _{k \rightarrow \infty} \frac{\rho_{k}}{k} \quad \text { and } \quad \frac{1}{\rho}=\inf _{k \geq 1} \frac{\lambda_{k}}{k}=\lim _{k \rightarrow \infty} \frac{\lambda_{k}}{k} .
$$

In particular, if $\Delta \neq \varnothing$, then $\rho>1$ and $\min \left\{k-\lambda_{k}, \rho_{k}-k\right\} \rightarrow \infty$ as $k \rightarrow \infty$.

Proof. The second part of the statement is trivial, and we can just focus on the limits. To this end, we have by Lemma 2.4 that $\lambda_{h+k} \leq \lambda_{h}+\lambda_{k} \leq \rho_{h}+\rho_{k} \leq \rho_{h+k}$ for all $h, k \in \mathbb{N}$.

So, applying Fekete's Lemma to the sequences $\left(\rho_{k}\right)_{k \geq 1}$ and $\left(-\lambda_{k}\right)_{k \geq 1}$, we find that the limits of $\frac{1}{k} \rho_{k}$ and $\frac{1}{k} \lambda_{k}$ as $k \rightarrow \infty$ exist and are equal, respectively, to $\varrho$ and $\lambda$, where for ease of notation we set

$$
\varrho:=\sup _{k \geq 1} \frac{1}{k} \rho_{k} \quad \text { and } \quad \lambda:=\inf _{k \geq 1} \frac{1}{k} \lambda_{k} .
$$

We are left to show that $\rho=\varrho$ and $1 / \rho=\lambda$.

CASE 1: $\rho=\infty$.

For each $\varepsilon \in \mathbb{R}^{+}$there exists $L_{\varepsilon} \in \mathscr{L}$ with $L_{\varepsilon}^{+} \neq \varnothing$ and $\rho\left(L_{\varepsilon}\right)>\frac{1}{\varepsilon}$. So, taking $k_{\varepsilon}=\min L_{\varepsilon}^{+}$yields that $\varrho \geq \frac{1}{k_{\varepsilon}} \rho_{k_{\varepsilon}} \geq \rho\left(L_{\varepsilon}\right)>\frac{1}{\varepsilon}$, whence $\varrho=\infty$. It remains to prove that $\lambda=0$. This is obvious if $\lambda_{k}=0$ for 
some $k \in \mathbb{N}$. Otherwise, we have from the above that $k_{\varepsilon}=\min L_{\varepsilon}$ (i.e., $L_{\varepsilon}=L_{\varepsilon}^{+}$) and there is $K_{\varepsilon} \in L_{\varepsilon}$ such that $K_{\varepsilon} / k_{\varepsilon} \geq \frac{1}{\varepsilon}$. Thus we find that

$$
0 \leq \lambda \leq \frac{\lambda_{K_{\varepsilon}}}{K_{\varepsilon}} \leq \frac{1}{K_{\varepsilon}} \min L_{\varepsilon} \leq \varepsilon
$$

which is enough to conclude that $\lambda=0$.

CASE 2: $\rho<\infty$.

If $\varrho>\rho$, then $\frac{1}{k} \rho_{k}>\rho$ for some $k \in \mathbb{N}$, and this in turn implies that there exists $\bar{L} \in \mathscr{L}_{k}$ with $\bar{L}^{+} \neq \varnothing$ such that $\rho<\frac{1}{k} \sup \bar{L} \leq \sup \bar{L} / \min \bar{L}^{+}=\rho(\bar{L})$, which is, however, a contradiction, because $\rho(\bar{L}) \leq \rho$. If, on the other hand, $\varrho<\rho$, then there is $\underline{L} \in \mathscr{L}$ with $\underline{L}^{+} \neq \varnothing$ and $\varrho<\rho(\underline{L})=\sup \underline{L} / \min \underline{L}^{+}$, and taking $k=\min \underline{L}^{+}$yields $\varrho<\frac{1}{k} \rho_{k} \leq \varrho$, which is still impossible. So we see that $\rho=\varrho$, and hence $\rho_{1}, \rho_{2}, \ldots$ are all finite. In particular, for each $k \in \mathbb{N}$ we can find a set $L_{k} \in \mathscr{L}_{k}$ such that $\rho_{k}=\max L_{k}$, and by Lemma 2.5(2) we have $\inf L_{k}=\min L_{k}=k$. It follows that $k \in \mathscr{U}_{\rho_{k}}$, and hence $\lambda_{\rho_{k}} \leq k$, for all $k \in \mathbb{N}$. Moreover,

$$
\lambda \geq \inf _{k \geq 1} \frac{\lambda_{k}}{\sup L_{k}}=\inf _{k \geq 1} \frac{1}{\rho\left(L_{k}\right)} \geq \frac{1}{\rho} .
$$

With this in mind, pick an integer $k \geq 1+\rho_{1}$ and let $l_{k}$ be the maximal $l \in \mathbb{N}$ such that $\rho_{l}<k$. Then $k=\rho_{l_{k}}+j_{k}$ for some $j_{k} \in \llbracket 1, \rho_{l_{k}+1}-\rho_{l_{k}} \rrbracket$, and since the function

$$
\left[0, \infty\left[\rightarrow \mathbb{R}, x \mapsto \frac{a+x}{b+x}\right.\right.
$$

is non-decreasing whenever $a, b \in \mathbb{R}^{+}$and $a \leq b$, we obtain from (2.3) and Lemma 2.4 that

$$
\frac{1}{\rho} \leq \lambda \leq \frac{\lambda_{k}}{k} \leq \frac{\lambda_{\rho_{l_{k}}}+\lambda_{j_{k}}}{\rho_{l_{k}}+j_{k}} \leq \frac{l_{k}+j_{k}}{\rho_{l_{k}}+j_{k}} \leq \frac{l_{k}+\rho_{l_{k}+1}-\rho_{l_{k}}}{\rho_{l_{k}}+\rho_{l_{k}+1}-\rho_{l_{k}}}=\frac{l_{k}}{\rho_{l_{k}+1}}+1-\frac{\rho_{l_{k}}}{\rho_{l_{k}+1}} .
$$

But the rightmost side of this last equation tends to $1 / \rho$ as $k \rightarrow \infty$, and hence $\lambda=1 / \rho$.

Proposition 2.8. Suppose that $\mathscr{L}$ has elasticity $\rho<\infty$. Then the following statements are equivalent:

(a) There is $L \in \mathscr{L}$ such that $L^{+} \neq \varnothing$ and $\rho(L)=\rho$.

(b) There exists $n \in \mathbb{N}$ such that $n k \rho=\rho_{n k}$ for all $k \in \mathbb{N}$.

(c) There is some $k \in \mathbb{N}$ such that $k \rho=\rho_{k}$.

Moreover, if at least one of conditions (a)-(c) is verified, there exists $K \in \mathbb{N}_{0}$ such that $\rho_{k+1} \leq \rho_{k}+K$ for every $k \in \mathbb{N}$.

Proof. Assume first that $\rho(L)=\rho$ for some $L \in \mathscr{L}$ with $L^{+} \neq \varnothing$, and set $n=\min L^{+}$. Since $\mathscr{L}$ is directed, it follows that for every $k \in \mathbb{N}$ there exists $L_{k} \in \mathscr{L}$ such that $n k \in k L \subseteq L_{k}$, and thus

$$
\frac{\rho_{n k}}{n k} \geq \frac{\sup L_{k}}{n k} \geq \frac{k \sup L}{n k}=\rho(L)=\rho \geq \frac{\rho_{n k}}{n k},
$$

where the last inequality comes as a consequence of Proposition 2.7. This proves that (a) $\Rightarrow(\mathrm{b})$.

Suppose now that (c) holds, and let $k \in \mathbb{N}$ such that $k \rho=\rho_{k}$. Then $\rho_{k}=\max L$ for some $L \in \mathscr{L}_{k}$ with $L^{+} \neq \varnothing$. So $k=\min L$ by Lemma $2.5(2)$, which gives $k \rho=\max L=k \rho(L)$, viz. $\rho(L)=\rho$. Putting it all together, we thus see that $(\mathrm{c}) \Rightarrow(\mathrm{a})$.

Since, on the other hand, it is obvious that $(b) \Rightarrow(c)$, we are just left to show the second part of the statement. To this end, let $n \in \mathbb{N}$ with the property that $n k \rho=\rho_{n k}$ for all $k \in \mathbb{N}$. 
Given $k \in \mathbb{N}$, there are $q_{k} \in \mathbb{N}_{0}$ and $r_{k} \in \llbracket 1, n \rrbracket$ such that $k=n q_{k}+r_{k}$, so we get from the above and Lemma 2.4(3) that

$$
k \rho-n(\rho-1) \leq\left(n q_{k}+r_{k}\right) \rho-r_{k}(\rho-1)=n q_{k} \rho+r_{k} \leq \rho_{n q_{k}}+\rho_{r_{k}} \leq \rho_{n q_{k}+r_{k}}=\rho_{k},
$$

namely $k \rho \leq \rho_{k}+n(\rho-1)$. It follows that

$$
\rho_{k+1} \leq(k+1) \rho \leq \rho_{k}+n(\rho-1)+\rho
$$

for every $k \in \mathbb{N}$, which completes the proof by taking $K=(n+1) \rho-n$.

Proposition 2.9. Let $\Delta^{\prime} \subseteq \Delta$ be a non-empty subset with $\operatorname{gcd}\left(\Delta^{\prime}\right) \leq \min \Delta$. Then $\operatorname{gcd}\left(\Delta^{\prime}\right)=\min \Delta$. In particular, gcd $\Delta=\min \Delta$.

Proof. Using that $\Delta^{\prime}$ is non-empty, define $\delta=\operatorname{gcd}\left(\Delta^{\prime}\right)$. There exist $\varepsilon_{1}, \ldots, \varepsilon_{n} \in\{ \pm 1\}, d_{1}, \ldots, d_{n} \in \Delta^{\prime}$, and $m_{1}, \ldots, m_{n} \in \mathbb{N}$ such that $\delta=\varepsilon_{1} m_{1} d_{1}+\ldots+\varepsilon_{n} m_{n} d_{n}$.

In addition, for each $i=1, \ldots, n$ we can find $x_{i} \in \mathbb{N}_{0}$ and $L_{i} \in \mathscr{L}$ such that $\left\{x_{i}, x_{i}+\varepsilon_{i} d_{i}\right\} \subseteq L_{i}$. Since $\mathscr{L}$ is directed, this gives that $\left\{m_{i} x_{i}, m_{i}\left(x_{i}+\varepsilon_{i} d_{i}\right)\right\} \subseteq m_{i} L_{i} \subseteq L_{i}^{\prime}$ for some $L_{i}^{\prime} \in \mathscr{L}$. Moreover, there is $L \in \mathscr{L}$ such that $L_{1}^{\prime}+\ldots+L_{n}^{\prime} \subseteq L$. Put $l=m_{1} x_{1}+\ldots+m_{n} x_{n}$.

Then we have by the above that $l+\delta=\sum_{i=1}^{n} m_{i}\left(x_{i}+\varepsilon_{i} d_{i}\right)$, and we see that both $l$ and $l+\delta$ belong to $L$. Thus $\min \Delta \leq \min \Delta(L) \leq \delta=\operatorname{gcd}\left(\Delta^{\prime}\right)$, and this is enough to conclude that $\operatorname{gcd}\left(\Delta^{\prime}\right)=\min \Delta$, as on the other hand we have by hypothesis that $\operatorname{gcd}\left(\Delta^{\prime}\right) \leq \min \Delta$.

The rest is clear, because $\Delta$ being non-empty implies that $\operatorname{gcd} \Delta \leq \min \Delta$.

Lemma 2.10. Let $d \in \Delta$.

(1) There exists $l \in \mathbb{N}_{0}$ such that, for every $q \in \mathbb{N}$, there is a set $L_{q} \in \mathscr{L}$ with $l q+d \cdot \llbracket 0, q \rrbracket \subseteq L_{q} \subseteq$ $\mathscr{U}_{l q} \cap \llbracket l q, \rho_{l q} \rrbracket$.

(2) Let $q \in \mathbb{N}$. Then for every sufficiently large $k \in \mathbb{N}$ there exists $L_{k} \in \mathscr{L}$ such that $k+d \cdot \llbracket 0, q \rrbracket \subseteq$ $L_{k} \subseteq \mathscr{U}_{k} \cap \llbracket k, \rho_{k} \rrbracket$.

Proof. Let $L_{0} \in \mathscr{L}$ and $l \in \mathbb{N}_{0}$ such that $\{l, l+d\}=L_{0} \cap \llbracket l, l+d \rrbracket$.

(1) Using that $\mathscr{L}$ is a directed family, we see that, for every $q \in \mathbb{N}$, there is a set $L \in \mathscr{L}$ such that $l q+d \cdot \llbracket 0, q \rrbracket=q\{l, l+d\} \subseteq q L_{0} \subseteq L \subseteq \mathscr{U}_{l q}$. The rest is obvious.

(2) Let $k$ be an integer $\geq l q+1$. Clearly, there exists $L^{\prime} \in \mathscr{L}$ such that $k-l q \in L^{\prime}$. On the other hand, it follows from the proof of point (1) that there is $L^{\prime \prime} \in \mathscr{L}$ with $l q+d \cdot \llbracket 0, q \rrbracket \subseteq L^{\prime \prime}$. Thus, we have that $k+d \cdot \llbracket 0, q \rrbracket \subseteq L^{\prime}+L^{\prime \prime} \subseteq L \subseteq \mathscr{U}_{k}$ for some $L \in \mathscr{L}$, and the rest is clear.

Lemma 2.11. Suppose $\Delta$ is finite non-empty, set $\delta=\min \Delta$, and let $k \in \mathbb{N}$.

(1) $\mathscr{U}_{k} \subseteq k+\delta \cdot \mathbb{Z}$ and $\Delta\left(\mathscr{U}_{k}\right) \subseteq \delta \cdot \mathbb{N}$.

(2) $\sup \Delta\left(\mathscr{U}_{k}\right) \leq \sup \Delta$.

Proof. (1) Proposition 2.9 implies that, for every $l \in \mathscr{U}_{k}, l-k$ is a multiple of $\delta$, and this yields that $\mathscr{U}_{k} \subseteq k+\delta \cdot \mathbb{Z}$. The rest is obvious.

(2) Assume to the contrary that $\sup \Delta\left(\mathscr{U}_{k}\right)>\sup \Delta$. Then $\mathscr{U}_{k} \neq \varnothing$ and there exist $l_{1}, l_{2} \in \mathbb{N}_{0}$ with $l_{2}-l_{1}>\sup \Delta$ and $\left\{l_{1}, l_{2}\right\}=\mathscr{U}_{k} \cap \llbracket l_{1}, l_{2} \rrbracket$. Accordingly, there also exist $L_{1}, L_{2} \in \mathscr{L}_{k}$ such that $l_{1} \in L_{1}$ and $l_{2} \in L_{2}$. We distinguish two cases.

CASE 1: $\min L_{2}=l_{2}$. 
Then $l_{1}<l_{2} \leq k$, and since $l_{1}, k \in L_{1}$, there exists $m_{1} \in L_{1}$ with $l_{1}<m_{1}$ and $\llbracket l_{1}, m_{1} \rrbracket \cap L_{1}=\left\{l_{1}, m_{1}\right\}$. But $L_{1} \subseteq \mathscr{U}_{k}$, so $l_{2} \leq m_{1}$, and hence $l_{2}-l_{1} \leq m_{1}-l_{1} \in \Delta\left(L_{1}\right) \subseteq \Delta$, viz. $l_{2}-l_{1} \leq \sup \Delta$, a contradiction.

CASE 2: $\min L_{2}<l_{2}$.

Then there is $m_{2} \in L_{2}$ with $m_{2}<l_{2}$ and $\llbracket m_{2}, l_{2} \rrbracket \cap L_{2}=\left\{m_{2}, l_{2}\right\}$. But $L_{2} \subseteq \mathscr{U}_{k}$, so we must have that $m_{2} \leq l_{1}$, and hence $l_{2}-l_{1} \leq l_{2}-m_{2} \in \Delta\left(L_{2}\right) \subseteq \Delta$, which is still a contradiction.

Lemma 2.12. Suppose $\Delta$ is finite non-empty and let $d \in \mathbb{N}$. Then the following statements are equivalent:

(a) There exists $M_{(a)} \in \mathbb{N}_{0}$ such that $\mathscr{U}_{k}$ is an AAP with difference d and bound $M_{(a)}$ for all sufficiently large $k$.

(b) There is $M_{(b)} \in \mathbb{N}_{0}$ such that $\mathscr{U}_{k} \cap \llbracket k-M_{(b)}, \rho_{k} \rrbracket$ is an AAP with difference $d$ and bound $M_{(b)}$ for all sufficiently large $k$.

(c) There exists $M_{(c)} \in \mathbb{N}_{0}$ such that $\mathscr{U}_{k} \cap \llbracket k, \rho_{k}-M_{(c)} \rrbracket$ is an AP with difference d for all sufficiently large $k$.

In addition, if at least one of conditions (a)-(c) is satisfied, then $d=\min \Delta$.

Proof. Set $\delta=\min \Delta$ for brevity's sake. Given $M \in \mathbb{N}_{0}$, we have by Proposition 2.7 and Lemma 2.10(2) that there exists $k_{M} \in \mathbb{N}$ such that

$$
k \geq \lambda_{k}+\max \{\delta, M\} \quad \text { and } \quad k+\delta \cdot \llbracket 0, M+1 \rrbracket \subseteq \mathscr{U}_{k} \cap \llbracket k, \rho_{k} \rrbracket \quad \text { for } k \geq k_{M} .
$$

It is thus clear that if at least one of conditions (a)-(c) is verified, then $d=\delta$, and this in turn makes it plain that (b) $\Leftrightarrow$ (c). Therefore it is enough to prove that (a) $\Leftrightarrow$ (c) with $d=\delta$.

(a) $\Rightarrow$ (c). Let $M_{(a)} \in \mathbb{N}_{0}$ and $k_{*} \in \mathbb{N}$ such that, for every $k \geq k_{*}, \mathscr{U}_{k}$ is an AAP with difference $\delta$ and bound $M_{(a)}$. Also, let $k_{a} \in \mathbb{N}$ such that (2.4) holds with $M=M_{(a)}$ and $k_{M}=k_{a}$.

Then, for each $k \geq \max \left\{k_{*}, k_{a}\right\}$ we have that $\mathscr{U}_{k}^{*}=\mathscr{U}_{k} \cap \llbracket \lambda_{k}+M_{(a)}, \rho_{k}-M_{(a)} \rrbracket$ is an AP with difference $\delta$ and, in addition, $k \in \mathscr{U}_{k}^{*}$. It is then evident that, for all sufficiently large $k$, also $\mathscr{U}_{k} \cap \llbracket k, \rho_{k}-M_{(a)} \rrbracket$ is an AP with difference $\delta$.

(c) $\Rightarrow$ (a). Let $M_{(c)} \in \mathbb{N}_{0}$ and $k_{c} \in \mathbb{N}$ be such that $\mathscr{U}_{k} \cap \llbracket k, \rho_{k}-M_{(c)} \rrbracket$ is an AP with difference $\delta$ for every $k \geq k_{c}$. Then set $M_{(a)}=\max \left\{k_{c}, M_{(c)}\right\}$ and let $k_{a} \in \mathbb{N}$ such that (2.4) is satisfied with $M=M_{(a)}$ and $k_{M}=k_{a}$.

Pick $k \geq \max \left\{k_{a}, k_{c}\right\}$ and $m \in \llbracket \lambda_{k}+M_{(a)}, k \rrbracket$ such that $k-m$ is a multiple of $\delta$. Clearly $k \leq \rho_{\lambda_{k}}$ and $\lambda_{k} \leq m-M_{(a)}$. So it follows from Lemma 2.4(3) that $k \leq \rho_{\lambda_{k}} \leq \rho_{m-M_{(a)}}$, and hence $k \leq \rho_{m}-M_{(a)}$, because

$$
k+M_{(a)} \leq \rho_{m-M_{(a)}}+M_{(a)} \leq \rho_{m-M_{(a)}}+\rho_{M_{(a)}} \leq \rho_{m} .
$$

On the other hand, $k \in m+\delta \cdot \mathbb{N}_{0}$ and $\mathscr{U}_{m} \cap \llbracket m, \rho_{m}-M_{(a)} \rrbracket$ is an AP with difference $\delta$, since $m \geq$ $\lambda_{k}+M_{(a)} \geq k_{c}$ and $M_{(a)} \geq M_{(c)}$, with the result that $\llbracket k, \rho_{k}-M_{(a)} \rrbracket \subseteq \llbracket k, \rho_{k}-M_{(c)} \rrbracket$. Putting it all together, we thus see that $k \in \mathscr{U}_{m}$, and hence $m \in \mathscr{U}_{k}$ by Lemma 2.4(1).

So, by the arbitrariness of $m \in \llbracket \lambda_{k}+M_{(a)}, k \rrbracket$ and the above, we find that

$$
\llbracket \lambda_{k}+M_{(a)}, \rho_{k}-M_{(a)} \rrbracket \cap(k+\delta \cdot \mathbb{Z})=\mathscr{U}_{k} \cap \llbracket \lambda_{k}+M_{(a)}, \rho_{k}-M_{(a)} \rrbracket,
$$

and this implies that $\mathscr{U}_{k}$ is an AAP with difference $\delta$ and bound $M_{(a)}$.

Lemma 2.13. Suppose $\mathscr{L}$ has elasticity $\rho<\infty$ and $\Delta$ is finite non-empty. 
(1) We have $\lambda_{k} \geq \rho^{-1} k>0$ for every $k \geq 1+\rho \max \Delta$.

(2) For $d \in \mathbb{N}$, the following statements are equivalent:

(a) $\mathscr{U}_{k}$ is an $A P$ with difference $d$ for all large $k$.

(b) $\mathscr{U}_{k} \cap \llbracket k, \rho_{k} \rrbracket$ is an AP with difference $d$ for all large $k$.

In addition, if either of conditions (a) and (b) holds, then $d=\min \Delta$.

Proof. (1) Let $k \geq 1+\rho \max \Delta$. There then exists $L \in \mathscr{L}$ such that $\lambda_{k}, k \in L$, and we have $k \leq \sup L \leq$ $\rho \min L^{+}$, that is $\min L^{+} \geq \rho^{-1} k>\max \Delta$. It follows that $L^{+}=L$, and hence $\lambda_{k}=\min L^{+} \geq \rho^{-1} k$, since $0 \in L$ would imply that $\max \Delta \geq \min L^{+}>\max \Delta$, which is impossible.

(2) We set $\delta=\min \Delta$. The last part of the claim ("In addition, if etc.") is clear from Lemma 2.12, and on the other hand it is obvious that $(\mathrm{a}) \Rightarrow(\mathrm{b})$. So we assume for the sequel that $d=\delta$ and we show that $(\mathrm{b}) \Rightarrow(\mathrm{a})$.

To start with, let $k_{0}^{\prime} \in \mathbb{N}$ such that $\lambda_{k} \geq \rho^{-1} k$ for $k \geq k_{0}^{\prime}$, which is possible by (1). Then let $k_{0}^{\prime \prime} \in \mathbb{N}_{0}$ such that $\mathscr{U}_{k} \cap \llbracket k, \rho_{k} \rrbracket$ is an $\mathrm{AP}$ for $k \geq k_{0}^{\prime \prime}$, and pick $k \geq \max \left\{k_{0}^{\prime}, \rho k_{0}^{\prime \prime}\right\}$. Since $k \in \mathscr{U}_{k}$, it is enough to prove that $\mathscr{U}_{k} \cap \llbracket \lambda_{k}, k \rrbracket$ is an $\mathrm{AP}$ with difference $\delta$.

To this end, let $m \in \llbracket \lambda_{k}, k \rrbracket$ such that $k-m$ is a multiple of $\delta$. Then $k_{0}^{\prime \prime} \leq \rho^{-1} k \leq \lambda_{k}$, and hence $\mathscr{U}_{m} \cap \llbracket m, \rho_{m} \rrbracket$ is an AP with difference $\delta$. But $m \leq k \leq \rho_{\lambda_{k}} \leq \rho_{m}$ and $k \in m+\delta \cdot \mathbb{N}_{0}$, so $k \in \mathscr{U}_{m}$, and this implies that $m \in \mathscr{U}_{k}$ by Lemma 2.4(1).

\section{Proof of Theorem 2.2.}

(1) Let $k$ be an arbitrary integer $\geq l q+1$.

(a) $\Rightarrow(\mathrm{b})$. By hypothesis, there are $M \in \mathbb{N}_{0}$ and $k_{0} \in \mathbb{N}$ such that $\mathscr{U}_{k} \cap \llbracket \lambda_{k}+M, \rho_{k}-M \rrbracket$ is an AP with difference $\delta$ for all $k \geq k_{0}$. On the other hand, we get from Proposition 2.7 that there also exists $k_{*} \in \mathbb{N}$ such that $k \geq \lambda_{k}+M$ and $\rho_{k-l q} \geq k$ for $k \geq k_{*}$. So, putting it all together, we see that $\mathscr{U}_{k} \cap \llbracket \rho_{k-l q}+l q, \rho_{k}-M \rrbracket$ is either empty or an AP with difference $\delta$ for all sufficiently large $k \in \mathbb{N}$.

(b) $\Rightarrow$ (a). We know from Proposition 2.9 that $\delta=\operatorname{gcd} \Delta$ and $\Delta \subseteq \delta \cdot \mathbb{N}$. In particular, this gives that $\mathscr{U}_{k} \subseteq \lambda_{k}+\delta \cdot \mathbb{N}_{0}$ and $q$ is a positive integer, since $q=\frac{1}{\delta} \max \Delta$ and $\max \Delta$ is a multiple of $\delta$. Moreover, $\mathscr{L}$ being a directed family implies that

$$
\mathscr{U}^{*}:=l q+\delta \cdot \llbracket 0, q \rrbracket \subseteq \mathscr{U}_{l q} .
$$

By Lemma 2.12, it is enough to show that there exists $k^{*} \in \mathbb{N}$ such that the set $\mathscr{U}_{k} \cap \llbracket k, \rho_{k}-M \rrbracket$ is an AP with difference $\delta$ for $k \geq k^{*}$. To this end, let

$$
\mathscr{U}^{\prime}=\mathscr{U}_{l q} \cap \llbracket \lambda_{l q}, l q-1 \rrbracket \text { and } \quad \mathscr{U}^{\prime \prime}=\mathscr{U}_{l q} \cap \llbracket(l+\delta) q+1, \rho_{l q} \rrbracket,
$$

and note that $\mathscr{U}^{\prime}, \mathscr{U}^{*}$, and $\mathscr{U}^{\prime \prime}$ are pairwise disjoint and $\mathscr{U}_{l q}=\mathscr{U}^{\prime} \cup \mathscr{U}^{*} \cup \mathscr{U}^{\prime \prime}$. It is evident that $k \in \mathscr{U}^{*}+\mathscr{U}_{k-l q}$, and Lemma 2.4(2) gives

$$
\mathscr{U}_{l q}+\mathscr{U}_{k-l q}=\left(\mathscr{U}^{\prime}+\mathscr{U}_{k-l q}\right) \cup\left(\mathscr{U}^{*}+\mathscr{U}_{k-l q}\right) \cup\left(\mathscr{U}^{\prime \prime}+\mathscr{U}_{k-l q}\right) \subseteq \mathscr{U}_{k} .
$$

On the other hand, we have by Lemma 2.11 that $\max \Delta\left(\mathscr{U}_{k-l q}\right) \leq \max \Delta$ and $\Delta\left(\mathscr{U}_{k-l q}\right) \subseteq \delta \cdot \mathbb{N}$.

It follows that $\mathscr{U}^{*}+\mathscr{U}_{k-l q}$ is an $\mathrm{AP}$ with difference $\delta$, and it is straightforward that $\sup \left(\mathscr{U}^{*}+\mathscr{U}_{k-l q}\right)=$ $l q+\delta q+\rho_{k-l q}$. Thus, if we let $k^{\prime} \in \mathbb{N}_{0}$ such that $\rho_{k} \geq M+k$ for $k \geq k^{\prime}$ (this is possible by Proposition 2.7) and we assume that there exists $k^{\prime \prime} \in \mathbb{N}$ such that $\mathscr{U}_{k} \cap \llbracket \rho_{k-l q}+l q, \rho_{k}-M \rrbracket$ is either empty or an AP 
with difference $\delta$ for $k \geq k^{\prime \prime}$, then it becomes clear from the above that $\mathscr{U}_{k} \cap \llbracket k, \rho_{k}-M \rrbracket$ is an AP with difference $\delta$ for $k \geq \max \left(k^{\prime}, k^{\prime \prime}\right)$.

(2) Note that $\mathbb{N} \cap L^{*} \neq \varnothing$, and set $n=\min \left(\mathbb{N} \cap L^{*}\right)$. Since $\rho<\infty$, we have by Proposition 2.8 that

$$
\sup L^{*}=\rho_{n} \quad \text { and } \quad \rho_{n k}=n k \rho \text { for all } k \in \mathbb{N} .
$$

Next, let $r$ be the smallest integer $>\rho \max \Delta$ such that $r \rho \in \mathbb{N}$ and $n r\left(\rho^{2}-1\right) \geq \frac{1}{\delta} \max \Delta$, which exists because $\rho$ is a rational number $>1$. We split the sequel of the proof into a series of claims.

Claim 1. Let $k$ be an integer $>\frac{1}{n} \rho \max \Delta$, and suppose that $k L^{*} \subseteq L$ for some $L \in \mathscr{L}$. Then $L=k L^{*}$ and $L$ is an AP with difference $\delta, \min L=n k$, and $\sup L=n k \rho$. In particular, $0 \notin L^{*}$.

Proof of Claim 1. Let $L \in \mathscr{L}$ such that $k L^{*} \subseteq L$. Then $n k \in L$, and since $n k>\rho \max \Delta$, we have by Lemma 2.13(1) that $\min L \geq 1$. On the other hand, $\rho<\infty$ implies by Proposition 2.7 that $\rho_{\kappa}<\infty$ for all $\kappa \in \mathbb{N}$, whence every set in $\mathscr{L}$ is finite. Accordingly, $\min L \leq n k$ and $k \sup L^{*} \leq \sup L<\infty$, with the result that

$$
\frac{\sup (L)}{\min L} \leq \rho=\rho\left(L^{*}\right)=\frac{1}{n} \sup L^{*}=\frac{k \sup L^{*}}{n k} \leq \frac{\sup (L)}{\min L} .
$$

So we see that $\min L=n k=\min \left(k L^{*}\right)$ and $\sup L=k \sup L^{*}=\sup \left(k L^{*}\right)$. But $k L^{*}$ is the $k$-fold sumset of an AP with difference $\delta$, so it is itself an AP with difference $\delta$. It follows that $L=k L^{*}$, and hence $\sup L=n k \rho$ by (2.7). The rest is clear.

Claim 2. $\mathscr{U}_{n r \rho}$ is an AP with difference $\delta$, and in addition $\lambda_{n r \rho}=n r$ and $\rho_{n r \rho}=n r \rho^{2}$.

Proof of Claim 2. Since $r \rho$ is a positive integer and $\mathscr{L}$ is a directed family, there exist $L_{1}, L_{2} \in \mathscr{L}$ such that $r L^{*} \subseteq L_{1}$ and $r \rho L^{*} \subseteq L_{2}$, which yields by (2.7) and Claim 1 that $L_{1}=r L^{*}$ and $L_{2}=r \rho L^{*}$, and both $L_{1}$ and $L_{2}$ are APs with difference $\delta$. So we get by (2.7) that

$$
\sup L_{1}=r \sup L^{*}=r \rho_{n}=n r \rho=r \rho \min L^{*}=\min L_{2},
$$

which yields $L_{1} \cup L_{2} \subseteq \mathscr{U}_{n r \rho}$. Since $L_{1} \cup L_{2}$ is an AP with difference $\delta$ and $\mathscr{U}_{n r \rho} \subseteq n r \rho+\delta \cdot \mathbb{Z}$ by Lemma $2.11(1)$, it is then enough for completing the proof of the claim to show that

$$
\lambda_{n r \rho}=\min L_{1}=r \min L^{*}=n r \quad \text { and } \quad \rho_{n r \rho}=\sup L_{2}=r \rho \sup L^{*}=n r \rho^{2},
$$

as in that case $L_{1} \cup L_{2}=\mathscr{U}_{n r \rho}$. Now, the latter equality is clear, because $\rho_{n r \rho}=n r \rho^{2}$ by (2.7). As for the former, suppose for a contradiction that $\min \mathscr{U}_{n r \rho}<n r$. Since (by construction) $n r \geq 1+\max \Delta$, there then exists $L \in \mathscr{L}_{n r \rho}$ such that $L^{+} \cap \llbracket 1, n r-1 \rrbracket$ is non-empty, with the result that $\rho\left(L^{+}\right) \geq n r \rho / \min L^{+}>\rho$, which is, however, impossible.

Claim 3. Given $j \in \llbracket 0, n-1 \rrbracket$, there exists $m_{j} \in \mathbb{N}$ such that the following conditions hold:

(i) $\rho_{m_{j} n+j}-m_{j} n \rho=\max _{\kappa \geq 1}\left(\rho_{n \kappa+j}-n \kappa \rho\right)$.

(ii) $\rho_{\left(m_{j}+i\right) n+j}=\rho_{m_{j} n+j}+i n \rho$ for all $i \in \mathbb{N}_{0}$.

Proof of Claim 3. By Proposition 2.7, $\rho_{\kappa} \leq \kappa \rho$ for every $\kappa \in \mathbb{N}$, and hence $\rho_{n \kappa+j}-n \kappa \rho \leq j \rho \leq(n-1) \rho$ for all $\kappa \in \mathbb{N}$. Since $\rho<\infty$, this gives that the set

$$
\mathcal{D}_{j}=\left\{\rho_{n \kappa+j}-n \kappa \rho: \kappa \in \mathbb{N}\right\} \subseteq \mathbb{Z}
$$


has a maximum element; let $m_{j} \in \mathbb{N}$ such that $\rho_{m_{j} n+j}-m_{j} n \rho=\max \mathcal{D}_{j}$. Accordingly, we get by (2.7) and Lemma 2.4(2) that, for every $i \in \mathbb{N}_{0}$,

$$
\begin{aligned}
\rho_{\left(m_{j}+i\right) n+j}-\left(m_{j}+i\right) n \rho & \leq \rho_{m_{j} n+j}-m_{j} n \rho \leq \rho_{m_{j} n+j}+\rho_{i n}-\left(m_{j}+i\right) n \rho \\
& \leq \rho_{\left(m_{j}+i\right) n+j}-\left(m_{j}+i\right) n \rho
\end{aligned}
$$

which leads to the desired conclusion.

At this point, let $k^{*}$ be a fixed integer $>m_{0}+\cdots+m_{n-1}+n r \rho$, where for each $j \in \llbracket 0, n-1 \rrbracket$ we denote by $m_{j}$ the smallest positive integer for which conditions (i) and (ii) in Claim 3 are verified. Then pick any integer $k \geq k^{*}$. By construction and (2.7), we have

$$
\sup \mathscr{U}_{k}=\rho_{k}=\rho_{k-n r \rho}+n r \rho^{2}=\sup \left(\mathscr{U}_{n r \rho}\right)+\sup \left(\mathscr{U}_{k-n r \rho}\right)=\sup \left(\mathscr{U}_{n r \rho}+\mathscr{U}_{k-n r \rho}\right)
$$

On the other hand, we get by Lemma 2.4(2) that $\mathscr{U}_{n r \rho}+\mathscr{U}_{k-n r \rho} \subseteq \mathscr{U}_{k}$, and by Claim 2 that $\mathscr{U}_{n r \rho}$ is an AP with difference $\delta$. Moreover, Claim 2 gives that

$$
\sup \mathscr{U}_{n r \rho}-\min \mathscr{U}_{n r \rho} \geq n r\left(\rho^{2}-1\right) \geq \frac{1}{\delta} \max \Delta
$$

while $\Delta\left(\mathscr{U}_{k-n r \rho}\right) \subseteq \delta \cdot \mathbb{N}_{0}$ and $\sup \Delta\left(\mathscr{U}_{k-n r \rho}\right) \leq \sup \Delta$ by Lemma 2.11. It follows that also the sumset $\mathscr{U}_{n r \rho}+\mathscr{U}_{k-n r \rho}$ is an AP with difference $\delta$, and so is $\mathscr{U}_{k} \cap \llbracket k, \rho_{k} \rrbracket$, as we see from the above that $\mathscr{U}_{k} \cap \llbracket k, \rho_{k} \rrbracket=$ $\left(\mathscr{U}_{n r \rho}+\mathscr{U}_{k-n r \rho}\right) \cap \llbracket k, \rho_{k} \rrbracket$. Therefore, we get by Lemma 2.13 that also $\mathscr{U}_{k}$ is an AP with difference $\delta$, which completes the proof of the theorem, as $k$ is any integer $\geq k^{*}$.

Proof of Corollary 2.3. By hypothesis, there exist $k_{0}, d \in \mathbb{N}$ and $M \in \mathbb{N}_{0}$ such that, for every $k \geq k_{0}, \mathscr{U}_{k}$ is an AAP with difference $d$ and bound $M$, with the result that the set $\mathscr{U}_{k}^{*}=\mathscr{U}_{k} \cap \llbracket \lambda_{k}+M, \rho_{k}-M \rrbracket$ is an AP with difference $d$. Moreover, we have that $k \in \mathscr{U}_{k}$ for all $k \in \mathbb{N}$, and by Lemma 2.12 we can assume $d=\delta$, so that $\mathscr{U}_{k}^{*} \subseteq k+\delta \cdot \mathbb{Z}$.

(1) Suppose now that $\rho_{k}<\infty$ for all $k \in \mathbb{N}$. Then, for every $k \in \mathbb{N}$ we can write

$$
\mathscr{U}_{k}=\left(\llbracket \lambda_{k}, k-1 \rrbracket \cap \mathscr{U}_{k}\right) \cup\{k\} \cup\left(\llbracket k+1, \rho_{k} \rrbracket \cap \mathscr{U}_{k}\right),
$$

which shows, together with the preliminary considerations, that all of $\mathscr{U}_{1}, \mathscr{U}_{2}, \ldots$ are AAPs with difference $d$ and bound $\max \left\{M, M_{0}\right\}$, where $M_{0}=\sup _{1 \leq k<k_{0}} \max \left\{k-\lambda_{k}, \rho_{k}-k\right\}$.

(2) If there is some $k \in \mathbb{N}$ such that $\rho_{k}=\infty$, then each side of equation (2.1) is infinite, and we are done. Otherwise, we have by the preliminary considerations that, for $k \geq k_{0}$,

$$
\frac{\left(\rho_{k}-M\right)-\left(\lambda_{k}+M\right)-\delta}{k \delta} \leq \frac{\left|\mathscr{U}_{k}\right|}{k} \leq \frac{\rho_{k}-\lambda_{k}+\delta}{k \delta},
$$

which, combined with Proposition 2.7, leads, in the limit as $k \rightarrow \infty$, to the desired conclusion that the rightmost and leftmost sides of (2.1) are equal.

As for the rest, if $X$ and $Y$ are non-empty sets of integers, then $|X+Y| \geq|X|+|Y|-1$ (see, e.g., [31, Theorem 3.1]). This, along with Lemma 2.4, yields that the sequence $\left(\left|\mathscr{U}_{k}\right|-1\right)_{k \geq 1}$ is superadditive, so we are done by Fekete's lemma. 


\section{Arithmetic of Commutative monoids}

In this section we apply the results on directed families from Section 2 (in particular, Theorem 2.2 and Corollary 2.3) to systems of sets of lengths of certain classes of semigroups. The main abstract results are Theorems 3.5 and 3.6, and they are valid for a class of commutative semigroups which are not necessarily cancellative. These theorems as well as the preparatory lemmas are well-known in the cancellative setting, partly with different bounds. However, there are several striking differences between the present setting and the cancellative one, which are gathered in Remarks 3.11. The semigroups we have in mind are certain semigroups of ideals and of modules. They will be discussed in Subsections 3.2 and 3.3 and have been the motivating examples for this paper (Corollaries 3.8 and 3.9).

We first introduce a suitable class of semigroups and give some motivation for it. A semigroup $H$ will be a non-empty set together with an associative and commutative binary operation such that $H$ possesses an identity element, which we denote by $1_{H}$ (or simply by 1 ) if $H$ is written multiplicatively. Apart from semigroups of modules (discussed in Subsection 3.3) and from power monoids (introduced in Subsection 3.4), we will always adopt the multiplicative notation. We use $H^{\times}$for the group of invertible elements of $H$, and if $H$ is cancellative, then we denote by $\mathrm{q}(H)$ the quotient group of $H$.

Suppose that $H$ has the property that every non-unit can be written as a finite product of distinguished elements. Our goal is to count the number of factors in such a factorization, and then to attach to each element $a \in H$ a set of factorization lengths $\mathrm{L}(a) \subseteq \mathbb{N}_{0}$. This should be done in a way that the product of two factorizations of elements $a$ and $b$ is a factorization of $a b$, which implies that $\mathrm{L}(a)+\mathrm{L}(b) \subseteq \mathrm{L}(a b)$. Every unit $u \in H$ is considered to have an empty factorization, and hence we define $\mathrm{L}(u)=\{0\}$. So if $a$ is a non-unit and $b$ is any element of the semigroup such that $a=a b$, then $\mathrm{L}(a)+\mathrm{L}(b) \subseteq \mathrm{L}(a)$. In particular, if $\mathrm{L}(a)$ is a finite non-empty set, this is possible only if $\mathrm{L}(b)=\{0\}$, and hence $b$ has to be a unit.

Thus a theory of sets of factorization lengths subjected to the above constraints is necessarily restricted to semigroups satisfying one of the next two equivalent conditions:

- If $a, u \in H$ and $a=a u$, then $u \in H^{\times}$.

- If $a, b, u, v \in H$ are such that $a=b u$ and $b=a v$, then $u, v \in H^{\times}$.

We refer to such semigroups as unit-cancellative, and for convenience we adopt the following convention:

Throughout, a monoid will always mean a commutative unit-cancellative semigroup.

Let $H$ be a monoid. Given $a, b \in H$, we say that $a$ and $b$ are associated (and write $a \simeq b$ ) if $a H^{\times}=b H^{\times}$, which, by unit-cancellativity, is equivalent to $a H=b H$. This defines a congruence on $H$, and $H_{\text {red }}=H / \simeq$ denotes the associated reduced monoid of $H$. The monoid $H$ is called reduced if $a, b \in H$ and $a \simeq b$ yield $a=b$. We say that $a$ divides $b$ (and write $a \mid b$ ) if $b \in a H$. A non-unit element $p \in H$ is said to be:

- irreducible (or an atom) if $a, b \in H$ and $p=a b$ imply $a \in H^{\times}$or $b \in H^{\times}$;

- prime if $a, b \in H$ and $p \mid a b$ imply $p \mid a$ or $p \mid b$.

The prime elements of a monoid are irreducible. We denote by $\mathcal{A}(H)$ the set of atoms of $H$. We say that $H$ is atomic if every non-unit is a finite product of atoms. For a set $P$, we denote by $\mathcal{F}(P)$ the free abelian monoid with basis $P$. An element $a \in \mathcal{F}(P)$ will be written as $a=\prod_{p \in P} p^{v_{p}(a)}$, where $v_{p}(a) \in \mathbb{N}_{0}$ for all $p \in P$ and $\mathrm{v}_{p}(a)=0$ for almost all $p \in P$, and we will denote by $|a|=\sum_{p \in P} \mathrm{v}_{p}(a) \in \mathbb{N}_{0}$ the length of $a$. Clearly, $P$ is the set of atoms of $\mathcal{F}(P)$ and every atom is prime. 
A monoid $H$ is said to be a Krull monoid if it is cancellative and one of the following equivalent conditions holds:

(a) $H$ is completely integrally closed and $v$-noetherian.

(b) There are a free abelian monoid $F$ and a homomorphism $\varphi: H \rightarrow F$, called a divisor homomorphism, with the property that if $a, b \in H$ and $\varphi(a) \mid \varphi(b)$ (in $F$ ), then $a \mid b$ (in $H$ ).

The theory of Krull monoids is presented in the monographs [32, 23]. We recall that an integral domain is a Krull domain if and only if its monoid of non-zero elements is Krull. Thus Condition (a) shows that every noetherian integrally closed domain is Krull (for more examples we refer to [20]).

It is well-known that the ascending chain condition (shortly, ACC) on principal ideals implies atomicity (in various classes of rings and semigroups). For the sake of completeness we provide a short proof of this and some related facts in the present setting.

Lemma 3.1. Let $H$ be a monoid.

(1) If $H$ satisfies the ACC on principal ideals, then $H$ is atomic.

(2) If $H$ is atomic, then for every set $P$ of pairwise non-associated primes of $H$ there is a submonoid $T \subseteq H$ such that $H=\mathcal{F}(P) T$.

Proof. (1) Assume to the contrary that the set

$$
\Omega=\{a H: a \in H \text { is not a product of finitely many atoms }\}
$$

is non-empty. Then it has a maximal element, say $a H$. Since $a$ is not an atom, there are non-units $b, c \in H$ such that $a=b c$. Thus we obtain that $a H \subseteq b H$ and $a H \subseteq c H$. Assume for a contradiction that $a H=b H$. Then there is an element $d \in H$ such that $b=a d$, and unit-cancellativity implies that $c \in H^{\times}$, a contradiction. Thus $a H \subsetneq b H$, and similarly $a H \subsetneq c H$. It follows by the maximality of $a H$ that $b$ and $c$ are finite products of atoms, and hence so is $a$, a contradiction.

(2) Let $T \subseteq H$ be the set of all $a \in H$ such that $p \nmid a$ for all $p \in P$. Then $H^{\times} \subseteq T$ and $T$ is a submonoid of $H$. Since $H$ is atomic, it follows that $H=\mathcal{F}(P) T$.

Suppose that $H$ is an atomic monoid. If $a=u_{1} \cdot \ldots \cdot u_{k}$ is a product of $k$ atoms, then $k$ is called the length of the factorization and the set $\mathrm{L}_{H}(a)=\mathrm{L}(a) \subseteq \mathbb{N}$ of all possible factorization lengths is called the set of lengths of $a$. It is convenient to take $\mathrm{L}(u)=\{0\}$ for all units $u \in H^{\times}$. Then

$$
\mathscr{L}(H)=\{\mathrm{L}(a): a \in H\}
$$

denotes the system of sets of lengths of $H$. If $H \neq H^{\times}, \mathscr{L}(H)$ is a directed family of subsets of $\mathbb{N}_{0}$, and we set $\Delta(H)=\Delta(\mathscr{L}(H)), \rho(H)=\rho(\mathscr{L}(H))$, and for every $k \in \mathbb{N}$,

$$
\mathscr{U}_{k}(H)=\mathscr{U}_{k}(\mathscr{L}(H)), \quad \rho_{k}(H)=\rho_{k}(\mathscr{L}(H)), \quad \text { and } \quad \lambda_{k}(H)=\lambda_{k}(\mathscr{L}(H)) \text {. }
$$

If $H=H^{\times}$, then for convenience we set $\Delta(H)=\varnothing, \rho(H)=1$, and for every $k \in \mathbb{N}, \mathscr{U}_{k}(H)=\{k\}$ and $\rho_{k}(H)=\lambda_{k}(H)=k$. We say that $H$ is a BF-monoid if every $L \in \mathscr{L}(H)$ is finite, and that $H$ has accepted elasticity if $\rho(H)=\rho(L)<\infty$ for some $L \in \mathscr{L}(H)$. All examples discussed in this paper are BF-monoids, and Proposition 3.3 provides a sufficient condition for a monoid to be a BF-monoid.

With this in mind, we now recall a couple more of arithmetical concepts, that is the catenary and tame degrees and the $\omega$-invariants of a monoid. 
To begin, we denote by $\mathrm{Z}(H)=\mathcal{F}\left(\mathcal{A}\left(H_{\text {red }}\right)\right)$ the factorization monoid of $H$, and by $\pi: \mathrm{Z}(H) \rightarrow H_{\text {red }}$, $u \mapsto u$ for all $u \in \mathcal{A}\left(H_{\text {red }}\right)$, the canonical epimorphism. For every $a \in H$, we let

$$
\mathrm{Z}_{H}(a)=\mathrm{Z}(a)=\pi^{-1}\left(a H^{\times}\right) \subseteq \mathrm{Z}(H)
$$

be the set of factorizations of $a$, and we have $\mathrm{L}(a)=\{|z|: z \in \mathrm{Z}(a)\} \subseteq \mathbb{N}_{0}$.

Let $z, z^{\prime} \in \mathrm{Z}(H)$. Then there exist $\ell, m, n \in \mathbb{N}_{0}$ and $u_{1}, \ldots, u_{\ell}, v_{1}, \ldots, v_{m}, w_{1}, \ldots, w_{n} \in \mathcal{A}\left(H_{\text {red }}\right)$ with $\left\{v_{1}, \ldots, v_{m}\right\} \cap\left\{w_{1}, \ldots, w_{n}\right\}=\varnothing$ such that

$$
z=u_{1} \cdot \ldots \cdot u_{\ell} v_{1} \cdot \ldots \cdot v_{m} \quad \text { and } \quad z^{\prime}=u_{1} \cdot \ldots \cdot u_{\ell} w_{1} \cdot \ldots \cdot w_{n} .
$$

We call $\mathrm{d}\left(z, z^{\prime}\right)=\max \{m, n\}$ the distance between $z$ and $z^{\prime}$. Given $a \in H$, the catenary degree $\mathrm{c}(a)$ of $a$ is then the smallest $N \in \mathbb{N}_{0} \cup\{\infty\}$ with the following property:

For any two factorizations $z, z^{\prime} \in \mathrm{Z}(a)$ there are factorizations $z_{0}, \ldots, z_{k}$ of $a$ with $z_{0}=z$ and $z_{k}=z^{\prime}$ such that $\mathrm{d}\left(z_{i-1}, z_{i}\right) \leq N$ for every $i \in \llbracket 1, k \rrbracket$.

Accordingly, we define the catenary degree of $H$ by

$$
\mathrm{c}(H)=\sup \{\mathrm{c}(a): a \in H\} \in \mathbb{N}_{0} \cup\{\infty\} .
$$

Next for every subset $I \subseteq H$, we let $\omega(H, I)$ be the smallest $N \in \mathbb{N}_{0} \cup\{\infty\}$ for which the following holds :

If $n \in \mathbb{N}$ and $a_{1}, \ldots, a_{n} \in H$ with $a_{1} \cdot \ldots \cdot a_{n} \in I$, then there exists a subset $\Omega \subseteq \llbracket 1, n \rrbracket$ such that $|\Omega| \leq N$ and $\prod_{\nu \in \Omega} a_{\nu} \in I$.

In particular, for every $a \in H$ we let $\omega(H, a)=\omega(H, a H)$, and we set

$$
\omega(H)=\sup \{\omega(H, u): u \in \mathcal{A}(H)\} \in \mathbb{N}_{0} \cup\{\infty\} .
$$

By convention, empty products are equal to $1 \in H$. Thus, if $a \in H^{\times}$, then $\omega(H, a)=0$, and if $p \in H$ is a prime, then $\omega(H, p)=1$.

Lastly, for every $u \in \mathcal{A}\left(H_{\text {red }}\right)$ we let $\mathrm{t}(H, u)$ denote the smallest $N \in \mathbb{N}_{0} \cup\{\infty\}$ such that:

Given $a \in H$ with $\mathbf{Z}(a) \cap u \mathbf{Z}(H) \neq \varnothing$ and $z \in \mathbf{Z}(a)$, there exists a factorization $z^{\prime} \in \mathbf{Z}(a) \cap u \mathbf{Z}(H)$ with $\mathrm{d}\left(z, z^{\prime}\right) \leq N$.

We say that $H$ is locally tame if $\mathrm{t}(H, u)<\infty$ for all $u \in \mathcal{A}\left(H_{\text {red }}\right)$, and (globally) tame if its tame degree

$$
\mathrm{t}(H)=\sup \left\{\mathrm{t}(H, u): u \in \mathcal{A}\left(H_{\text {red }}\right)\right\} \in \mathbb{N}_{0} \cup\{\infty\}
$$

is finite. In the next proposition we collect some elementary properties of the above invariants that are well-known for cancellative monoids ([24, 25]).

Proposition 3.2. Let $H$ be an atomic monoid.

(1) For every $a \in H$ we have $\sup \mathrm{L}(a) \leq \omega(H, a)$. In particular, if $\omega(H, a)<\infty$ for every $a \in H$, then $H$ is a BF-monoid.

(2) For every $a \in H$ with $|\mathrm{Z}(a)| \geq 2$, we have $1+\sup \Delta(\mathrm{L}(a)) \leq \mathrm{c}(a)$. In particular, if $\Delta(H) \neq \varnothing$, then $1+\sup \Delta(H) \leq \mathrm{c}(H)$.

(3) For every $u \in \mathcal{A}(H)$ with $\omega(H, u)<\infty$ we have $\mathrm{t}(H, u) \leq \rho_{\omega(H, u)}(H)$. 
Proof. We may suppose that $H$ is reduced.

(1) Let $a \in H$. If $\omega(H, a)=\infty$, the assertion is trivial. Suppose that $\omega(H, a)<\infty$ and assume for a contradiction that $\sup \mathrm{L}(a)>\omega(H, a)$. Then there exist $m \in \mathbb{N}$ with $m>\omega(H, a)$ and atoms $v_{1}, \ldots, v_{m}$ such that $a=v_{1} \cdot \ldots \cdot v_{m}$. Accordingly, there is a subset $\Omega \subseteq \llbracket 1, m \rrbracket$, say $\Omega=\llbracket 1, \ell \rrbracket$ with $\ell \leq \omega(H, a)<m$, such that $a$ divides $v_{1} \cdot \ldots \cdot v_{\ell}$. Then unit-cancellativity implies that $v_{\ell+1} \cdot \ldots \cdot v_{m} \in H^{\times}$, a contradiction.

(2) We start by verifying a basic inequality. Let $a \in H$, and let $z, z^{\prime} \in \mathrm{Z}(a)$ be distinct factorizations. Set $x=\operatorname{gcd}\left(z, z^{\prime}\right)$, and $z=x y, z^{\prime}=x y^{\prime}$ with $y, y^{\prime} \in \mathbf{Z}(H)$. Then $\mathrm{d}\left(z, z^{\prime}\right)=\max \left\{|y|,\left|y^{\prime}\right|\right\}$, and unitcancellativity implies that $|y| \geq 1$ and $\left|y^{\prime}\right| \geq 1$. Thus it follows that

$$
1+|| z|-| z^{\prime}||=1+|| y|-| y^{\prime}|| \leq \max \left\{|y|,\left|y^{\prime}\right|\right\}=\mathrm{d}\left(z, z^{\prime}\right) .
$$

Now suppose that $a \in H$ with $\Delta(\mathrm{L}(a)) \neq \varnothing$ and let $s \in \Delta(\mathrm{L}(a))$. We have to show that $1+s \leq \mathrm{c}(a)$. Indeed, there exist $z, z^{\prime} \in \mathbf{Z}(a)$ such that $\left|z^{\prime}\right|=|z|+s$, but there is no factorization $z^{\prime \prime} \in \mathrm{Z}(a)$ with $|z|<\left|z^{\prime \prime}\right|<\left|z^{\prime}\right|$. On the other hand, there are factorizations $z_{0}, \ldots, z_{k}$ of $a$ with $z_{0}=z$ and $z_{k}=z^{\prime}$ such that $\mathrm{d}\left(z_{i-1}, z_{i}\right) \leq \mathrm{c}(a)$ for every $i \in \llbracket 1, k \rrbracket$. Thus there is some $\nu \in \llbracket 1, k \rrbracket$ such that $\left|z_{\nu-1}\right| \leq|z|$ and $\left|z_{\nu}\right| \geq\left|z^{\prime}\right|$, and hence we get from the above that

$$
1+s \leq 1+\left|z_{\nu}\right|-\left|z_{\nu-1}\right| \leq \mathrm{d}\left(z_{\nu-1}, z_{\nu}\right) \leq \mathrm{c}(a) .
$$

Finally, suppose that $\Delta(H) \neq \varnothing$. Then there is an element $a \in H$ with $|\mathrm{Z}(a)| \geq|\mathrm{L}(a)| \geq 2$, and for all $a \in H$ with $|\mathrm{Z}(a)| \geq 2$ we have $1+\sup \Delta(\mathrm{L}(a)) \leq \mathrm{c}(a) \leq \mathrm{c}(H)$. So the assertion follows.

(3) Pick $u \in \mathcal{A}(H)$ with $\omega(H, u)<\infty$, and let $a \in H$ with $\mathrm{Z}(a) \cap u \mathrm{Z}(H) \neq \varnothing$. If $z=v_{1} \cdot \ldots \cdot v_{n} \in \mathrm{Z}(a)$, then $u \mid v_{1} \cdot \ldots \cdot v_{n}$ (in $H$ ), so (after renumbering if necessary) there exists $k \in \llbracket 1, n \rrbracket$ with $k \leq \omega(H, u)$ such that $u \mid v_{1} \cdot \ldots \cdot v_{k}$. Thus $v_{1} \cdot \ldots \cdot v_{k}=u u_{2} \cdot \ldots \cdot u_{\ell}$ for some $u_{2}, \ldots, u_{\ell} \in \mathcal{A}(H)$ with $\ell \leq \rho_{k}(H) \leq \rho_{\omega(H, u)}(H)$. It follows that $z^{\prime}=u u_{2} \cdot \ldots \cdot u_{\ell} \cdot v_{k+1} \cdot \ldots \cdot v_{n} \in \mathrm{Z}(a)$ and $\mathrm{d}\left(z, z^{\prime}\right) \leq \max \{k, \ell\} \leq \rho_{\omega(H, u)}(H)$. Therefore $\mathrm{t}(H, u) \leq \rho_{\omega(H, u)}(H)$.

Next we study the $\omega$-invariants with ideal theoretic tools. A weak ideal system on a monoid $H$ is a map $r: \mathscr{P}(H) \rightarrow \mathscr{P}(H), X \mapsto X_{r}$ such that the following conditions are satisfies for all subsets $X, Y \subseteq H$ and all $c \in H$ :

- $X \subseteq X_{r}$.

- $X \subseteq Y_{r}$ implies $X_{r} \subseteq Y_{r}$.

- $c H \subseteq\{c\}_{r}$.

- $c X_{r} \subseteq(c X)_{r}$.

An ideal system on $H$ is a weak ideal system such that $c X_{r}=(c X)_{r}$ for all $X \subseteq H$ and all $c \in H$. We refer to [32] for a thorough treatment of ideal systems.

Let $r$ be a weak ideal system. A subset $I \subseteq H$ is called an $r$-ideal if $I_{r}=I$. We denote by $\mathcal{I}_{r}(H)$ the set of all non-empty $r$-ideals, and we define $r$-multiplication by setting $I \cdot_{r} J=(I J)_{r}$ for all $I, J \in \mathcal{I}_{r}(H)$. Then $\mathcal{I}_{r}(H)$ together with $r$-multiplication is a reduced semigroup with identity element $H$. An $r$-ideal $\mathfrak{p} \in \mathcal{I}_{r}(H)$ is called prime if $\mathfrak{p} \neq H$, and $a, b \in H$ and $a b \in \mathfrak{p}$ imply $a \in \mathfrak{p}$ or $b \in \mathfrak{p}$. We denote by $r$-spec $(H)$ the set of all prime $r$-ideals of $H$. The monoid $H$ is called $r$-noetherian if it satisfies the ACC on $r$-ideals. For $I \in \mathcal{I}_{r}(H)$ and $h \in H$, we set $(I: h)=\{b \in H: h b \in I\}$ and note that $(I: h) \in \mathcal{I}_{r}(H)$ (see [32, Section $2.4])$. 
The result of the next proposition was first established for the $v$-system (i.e., the system of divisorial ideals) with a different proof (see [24, Theorem 4.2]).

Proposition 3.3. Let $H$ be a monoid and let $r$ be an $r$-noetherian weak ideal system on $H$.

(1) For every $I \in \mathcal{I}_{r}(H)$ we have $\omega(H, I)<\infty$.

(2) If principal ideals are $r$-ideals, then $H$ is a BF-monoid.

Proof. (1) If $I \subseteq H$ is a prime ideal, then $\omega(H, I)=1$. We start with the following two assertions.

(A1) Given $I \in \mathcal{I}_{r}(H)$, there exists $J \in \mathcal{I}_{r}(H)$ with $I \subsetneq J$ such that the set $(I: h)$ is a prime ideal for every $h \in J \backslash I$.

(A2) If $I, J \in \mathcal{I}_{r}(H)$ are as above, then $\omega(H, I) \leq \omega(H, J)+1$.

Proof of (A1). Consider the set

$$
\Omega=\{(I: h): h \in H \backslash I\} \subseteq \mathcal{I}_{r}(H)
$$

and choose an element $h_{0} \in H \backslash I$ such that $\mathfrak{p}=\left(I: h_{0}\right) \in \Omega$ is maximal. We claim that $\mathfrak{p}$ is a prime ideal. In fact, assume that there exist $c, d \in H$ with $c d \in \mathfrak{p}$, but $d \notin \mathfrak{p}$. Then $h_{0} c d \in I, h_{0} d \notin I$, and

$$
\mathfrak{p}=\left(I: h_{0}\right) \subsetneq\left(I: h_{0} c\right) \text {. }
$$

Thus, the maximality of $\mathfrak{p}$ yields that $h_{0} c \in I$, and hence $c \in \mathfrak{p}$. Now, we assert that the ideal

$$
J=\left(I \cup\left\{h_{0}\right\}\right)_{r}
$$

has the required properties. Indeed, let $h \in J \backslash I$, and let $b \in H$ with $h_{0} b \in I$. Since $I b \subseteq I$, we have

$$
h b \in J b=\left(I \cup\left\{h_{0}\right\}\right)_{r} b \subseteq\left(b I \cup\left\{h_{0} b\right\}\right)_{r} \subseteq I_{r}=I .
$$

This shows that

$$
\mathfrak{p}=\left(I: h_{0}\right) \subseteq(I: h),
$$

which, again by the maximality of $\mathfrak{p}$, implies that $\mathfrak{p}=(I: h)$ is a prime ideal.

Proof of (A2). Let $n \in \mathbb{N}$ and $a_{1}, \ldots, a_{n} \in H$ with $a_{1} \cdot \ldots \cdot a_{n} \in I$. Then $a_{1} \cdot \ldots \cdot a_{n} \in J$, and there is a subset $\Omega \subseteq \llbracket 1, n \rrbracket$, say $\Omega=\llbracket 1, m \rrbracket$, with $m \leq \omega(H, J)$ and $a_{1} \cdot \ldots \cdot a_{m} \in J$. If $a_{1} \cdot \ldots \cdot a_{m} \in I$, then we are done. Otherwise, $a_{m+1} \cdot \ldots \cdot a_{n} \in\left(I: a_{1} \cdot \ldots \cdot a_{m}\right)=\mathfrak{p}$, and since $\mathfrak{p}$ is a prime ideal, there exists $\nu \in \llbracket m+1, n \rrbracket$, say $\nu=m+1$, such that $a_{m+1} \in \mathfrak{p}$. Clearly, this implies that $a_{1} \cdot \ldots \cdot a_{m+1} \in I$, and hence $\omega(H, I) \leq \omega(H, J)+1$.

Let $I \in \mathcal{I}_{r}(H)$. Since $H$ is $r$-noetherian, we get from (A1) that there is an ascending chain of $r$-ideals

$$
I=I_{0} \subsetneq I_{1} \subsetneq I_{2} \subsetneq \ldots \subsetneq I_{n}=H
$$

such that $\left(I_{\nu-1}: h\right)$ is a prime ideal for all $\nu \in \llbracket 1, n \rrbracket$ and $h \in I_{\nu} \backslash I_{\nu-1}$. So, we conclude by (A2) that

$$
\omega(H, I) \leq \omega\left(H, I_{1}\right)+1 \leq \omega\left(H, I_{2}\right)+2 \leq \ldots \leq \omega\left(H, I_{n}\right)+n=n .
$$

(2) Since $H$ is $r$-noetherian, $H$ satisfies the ACC on principal ideals, and hence it is atomic by Lemma 3.1. Thus, the assertion follows by point (1) and Proposition 3.2(1). 
In the next proposition we will need the $s$-system of a monoid. Indeed, if $H$ is a monoid, then the $s$-system $s: \mathscr{P}(H) \rightarrow \mathscr{P}(H)$, defined by $X_{s}=X H$ for all $X \subseteq H$, is an ideal system on $H$.

Proposition 3.4. Let $H$ be a monoid such that $H_{\text {red }}$ is finitely generated. Then $H$ is an s-noetherian BF-monoid, and the following hold:

(1) $\rho(H) \in \mathbb{Q}$ and there exists $K \in \mathbb{N}$ such that $\rho_{k+1}(H) \leq \rho_{k}(H)+K<\infty$ for all $k \in \mathbb{N}$.

(2) The set of distances $\Delta(H)$ is finite.

(3) $\omega(H)<\infty$ and $\mathrm{t}(H)<\infty$.

Proof. The monoid $H$ is s-noetherian by [32, Theorem 3.6] and hence it is a BF-monoid by Proposition 3.3. We are left to verify the assertion on the arithmetical invariants, and to do so we may assume without restriction that $H$ is reduced and $\mathcal{A}(H)=\left\{u_{1}, \ldots, u_{s}\right\}$ is non-empty. We will denote by $\preceq$ the product order induced on $\mathbb{N}_{0}^{s}$ by the usual order of $\mathbb{N}_{0}$.

(1) Let $\pi: \mathrm{Z}(H) \rightarrow H$ be the canonical epimorphism and set $S^{*}=S \backslash\{(1,1)\}$, where

$$
S=\{(x, y) \in \mathbf{Z}(H) \times \mathbf{Z}(H): \pi(x z)=\pi(y z) \text { for some } z \in \mathbf{Z}(H)\} .
$$

Pick $(x, y) \in S^{*}$ with $x \neq 1$, and suppose for a contradiction that $y=1$. Then $\pi(y)=1$, and letting $z \in$ $\mathrm{Z}(H)$ such that $\pi(x z)=\pi(y z)$ yields $\pi(z) \pi(x)=\pi(z)$. But this implies, together with unit-cancellativity, that $\pi(x)=1$, and hence $x=1$, a contradiction.

It follows that $|x| \geq 1$ and $|y| \geq 1$ for all $(x, y) \in S^{*}$, and accordingly we can define

$$
\rho^{*}(H)=\sup \left\{|y|^{-1}|x|:(x, y) \in S^{*}\right\} .
$$

We note that $\rho(H) \leq \rho^{*}(H)$, and we want to show that there exists $(\bar{x}, \bar{y}) \in S^{*}$ such that $\rho^{*}(H)=|\bar{y}|^{-1}|\bar{x}|$. To this end, consider the homomorphism

$$
f: \mathbf{Z}(H) \times \mathbf{Z}(H) \rightarrow\left(\mathbb{N}_{0}^{s} \times \mathbb{N}_{0}^{s},+\right),\left(\prod_{i=1}^{s} u_{i}^{m_{i}}, \prod_{i=1}^{s} u_{i}^{n_{i}}\right) \mapsto\left(\left(m_{i}\right)_{i=1}^{s},\left(n_{i}\right)_{i=1}^{s}\right) .
$$

In fact, $f$ is an isomorphism, and we have by Dickson's Theorem [23, Theorem 1.5.3] that the set $f\left(S^{*}\right)$ has only finitely many minimal points relative to the order $\preceq$. Let $T \subseteq S^{*}$ be the inverse image of the set of these minimal points. It is sufficient to prove that

$$
\frac{|x|}{|y|} \leq \max \left\{\frac{\left|x^{\prime}\right|}{\left|y^{\prime}\right|}:\left(x^{\prime}, y^{\prime}\right) \in T\right\} \quad \text { for all } \quad(x, y) \in S^{*} .
$$

For this, pick $(x, y) \in S^{*}$. We proceed by induction on $|x|+|y|$. If $(x, y) \in T$, then there is nothing to do. So suppose that $(x, y) \notin T$. Then there exists $\left(x_{1}, y_{1}\right) \in T$ such that $(x, y)=\left(x_{1} x_{2}, y_{1} y_{2}\right)$ for some $\left(x_{2}, y_{2}\right) \in \mathbf{Z}(H) \times \mathbf{Z}(H)$. We claim that $\left(x_{2}, y_{2}\right) \in S^{*}$.

Indeed, let $z, z_{1} \in \mathbf{Z}(H)$ such that $\pi(x z)=\pi(y z)$ and $\pi\left(x_{1} z_{1}\right)=\pi\left(y_{1} z_{1}\right)$, and set $z_{2}=x_{1} y_{1} z_{1} z$. Then $x_{2} z_{2}=x z y_{1} z_{1}$ and $y_{2} z_{2}=y z x_{1} z_{1}$, which implies that $\pi\left(x_{2} z_{2}\right)=\pi\left(y_{2} z_{2}\right)$, and hence $\left(x_{2}, y_{2}\right) \in S^{*}$.

It is thus clear that $\left|x_{j}\right|+\left|y_{j}\right|<|x|+|y|$ for $j \in\{1,2\}$, and by the induction hypothesis we have that

$$
\frac{|x|}{|y|}=\frac{\left|x_{1}\right|+\left|x_{2}\right|}{\left|y_{1}\right|+\left|y_{2}\right|} \leq \max \left\{\frac{\left|x_{1}\right|}{\left|y_{1}\right|}, \frac{\left|x_{2}\right|}{\left|y_{2}\right|}\right\} \leq \max \left\{\frac{\left|x^{\prime}\right|}{\left|y^{\prime}\right|}:\left(x^{\prime}, y^{\prime}\right) \in T\right\},
$$

as was desired. 
Now, based on the above, let $(\bar{x}, \bar{y}) \in T$ such that $\rho^{*}(H)=|\bar{y}|^{-1}|\bar{x}|$, and let $\bar{z} \in \mathbf{Z}(H)$ such that $\pi(\bar{x} \bar{z})=\pi(\bar{y} \bar{z})$. Then, for every $i \in \mathbb{N}$ we have $\pi\left(\bar{x}^{i} \bar{z}\right)=\pi\left(\bar{y}^{i} \bar{z}\right)$, and setting $a_{i}=\pi\left(\bar{x}^{i} \bar{z}\right)$ gives that

$$
\rho\left(\mathrm{L}\left(a_{i}\right)\right)=\frac{\max \mathrm{L}\left(a_{i}\right)}{\min \mathrm{L}\left(a_{i}\right)} \geq \frac{\left|\bar{x}^{i} \bar{z}\right|}{\left|\bar{y}^{i} \bar{z}\right|}=\frac{i|\bar{x}|+|\bar{z}|}{i|\bar{y}|+|\bar{z}|} .
$$

It follows that

$$
\rho(H) \geq \lim _{i \rightarrow \infty} \frac{i|\bar{x}|+|\bar{z}|}{i|\bar{y}|+|\bar{z}|}=\frac{|\bar{x}|}{|\bar{y}|}=\rho^{*}(H) \geq \rho(H),
$$

whence $\rho(H)=\rho^{*}(H)=|\bar{y}|^{-1}|\bar{x}| \in \mathbb{Q}$. In particular, $\rho(H)$ is finite, which implies by Proposition 2.7 that $\rho_{k}(H) \leq k \rho(H)<\infty$ for all $k \in \mathbb{N}$.

We are left to show that there exists $K \in \mathbb{N}$ such that $\rho_{k+1}(H)-\rho_{k}(H) \leq K$ for all $k \in \mathbb{N}$. To this end, set $n=|\bar{x}|$ and $m=|\bar{y}|$. Since $\pi\left(\bar{x}^{i} \bar{z}\right)=\pi\left(\bar{y}^{i} \bar{z}\right)$ and $\rho(H)=\left|\bar{y}^{i}\right|{ }^{-1}\left|\bar{x}^{i}\right|$ for all $i \in \mathbb{N}$, we may assume that $|\bar{z}| \leq m$, otherwise we replace $\bar{x}$ with $\bar{x}^{|\bar{z}|}$ and $\bar{y}$ with $\bar{y}^{|\bar{z}|}$ (we use here that $|\bar{x}| \geq 1$ and $|\bar{y}| \geq 1$ ). Moreover, for every $i \in \mathbb{N}$ we have

$$
\rho_{i m+|z|}(H) \geq i n+|z|=i m \rho(H)+|z|,
$$

whence

$$
(i m+|z|) \rho(H)-m(\rho(H)-1) \leq(i m+|z|) \rho(H)-|z|(\rho(H)-1)=i m \rho(H)+|z| \leq \rho_{i m+|z|}(H),
$$

and thus

$$
(i m+|z|) \rho(H)-\rho_{i m+|z|}(H) \leq m(\rho(H)-1) .
$$

Now let $k \geq|z|+1$. Then there is an $i \in \mathbb{N}$ such that

$$
(i-1) m+|z| \leq k-1<k \leq i m+|z| .
$$

Thus we obtain that

$$
\begin{aligned}
\rho_{k}(H)-\rho_{k-1}(H) & \leq \rho_{i m+|z|}(H)-\rho_{(i-1) m+|z|}(H) \\
& \leq(i m+|z|) \rho(H)+m(\rho(H)-1)-((i-1) m+|z|) \rho(H) \\
& =m(2 \rho(H)-1) .
\end{aligned}
$$

(2) Set

$$
M=\left\{\left(m_{1}, \ldots, m_{s}\right) \in \mathbb{N}_{0}^{s}: \prod_{i=1}^{s} u_{i}^{m_{i}} \text { has a factorization of length larger than } m_{1}+\ldots+m_{s}\right\} \subseteq \mathbb{N}_{0}^{s} .
$$

By Dickson's theorem [23, Theorem 1.5.3], we have that the set $\operatorname{Min}(M)$ of minimal points of $M$ (relative to the order $\preceq)$ is finite. For each $\boldsymbol{m} \in \operatorname{Min}(M)$, we thus choose an $\boldsymbol{m}^{*}=\left(m_{1}^{*}, \ldots, m_{s}^{*}\right) \in \mathbb{N}_{0}^{s}$ such that $|\boldsymbol{m}|<\left|\boldsymbol{m}^{*}\right|$ and $\prod_{i=1}^{s} u_{i}^{m_{i}}=\prod_{i=1}^{s} u_{i}^{m_{i}^{*}}$. Accordingly, we set

$$
K=\max \left\{\left|\boldsymbol{m}^{*}\right|-|\boldsymbol{m}|: \boldsymbol{m} \in \operatorname{Min}(M)\right\} .
$$

Clearly, it is enough to show that for every $a \in H$ and every $k \in \mathrm{L}(a)$ with $k<\max \mathrm{L}(a)$ there exists $\ell \in \mathrm{L}(a)$ with $k<\ell \leq k+K$.

Let $a=u_{1}^{k_{1}} \cdot \ldots \cdot u_{s}^{k_{s}} \in H$ with $k=k_{1}+\ldots+k_{s}<\max \mathrm{L}(a)$. Then $\boldsymbol{k}=\left(k_{1}, \ldots, k_{s}\right) \in M$, and hence there is an $\boldsymbol{m}=\left(m_{1}, \ldots, m_{s}\right) \in \operatorname{Min}(M)$ with $\boldsymbol{m} \leq \boldsymbol{k}$. Then

$$
a=u_{1}^{k_{1}} \cdot \ldots \cdot u_{s}^{k_{s}}=u_{1}^{m_{1}} \cdot \ldots \cdot u_{s}^{m_{s}} \cdot u_{1}^{k_{1}-m_{1}} \cdot \ldots \cdot u_{s}^{k_{s}-m_{s}}=u_{1}^{m_{1}^{*}} \cdot \ldots \cdot u_{s}^{m_{s}^{*}} \cdot u_{1}^{k_{1}-m_{1}} \cdot \ldots \cdot u_{s}^{k_{s}-m_{s}}
$$


has a factorization of length $\left|\boldsymbol{m}^{*}\right|-|\boldsymbol{m}|+k$ with $k<\left|\boldsymbol{m}^{*}\right|-|\boldsymbol{m}|+k \leq k+K$.

(3) Proposition 3.3 implies that $\omega(H, u)<\infty$ for all $u \in \mathcal{A}(H)$. It follows that $\omega(H)<\infty$, because $\mathcal{A}(H)$ is finite. Together with Proposition 3.2(3) and point (1) above, this yields that $\mathrm{t}(H, u) \leq$ $\rho_{\omega(H, u)}(H) \leq \rho_{\omega(H)}(H)<\infty$ for every $u \in \mathcal{A}(H)$, and hence $\mathrm{t}(H)<\infty$.

Theorem 3.5. Let $H$ be an atomic monoid.

(1) Suppose that $\mathrm{c}(H)<\infty$ and $H$ has accepted elasticity. Then the set of distances $\Delta(H)$ is finite and there is a $K \in \mathbb{N}_{0}$ such that $\rho_{k+1}(H) \leq \rho_{k}(H)+K$ for all $k \in \mathbb{N}$.

(2) If $H$ is cancellative and $\omega(H)<\infty$, then $\mathrm{c}(H) \leq \mathrm{t}(H) \leq \omega(H)^{2}, \rho(H) \leq \omega(H)$, and $\rho_{k+1}(H) \leq$ $\rho_{k}(H)+\max \{1, \omega(H)-1\}$ for all $k \in \mathbb{N}$.

In both cases, $\mathscr{L}(H)$ satisfies the Structure Theorem for Unions.

Proof. If the statements on the invariants hold true, then in both cases Theorem 2.2 shows that $\mathscr{L}(H)$ satisfies the Structure Theorem for Unions (indeed, Condition (1)(b) of Theorem 2.2 holds by the remark following Theorem 2.2).

(1) We have that $\Delta(H)$ is finite by Proposition 3.2(2), while Proposition 2.8 implies the existence of a $K \in \mathbb{R}$ with the required properties.

(2) The inequalities follow from [25, Propositions 3.5 and 3.6].

Theorem 3.6. Let $H$ be a monoid such that $H=\mathcal{F}(P) \times T$, where $P \subseteq H$ is a set of pairwise nonassociated primes and $T \subseteq H$ is a submonoid such that $T_{\text {red }}$ is finitely generated.

(1) The set of distances $\Delta(H)$ and the invariants $\rho(H), \omega(H)$, and $\mathrm{t}(H)$ are all finite.

(2) $\mathscr{L}(H)$ satisfies the Structure Theorem for Unions.

Proof. Proposition 3.4(3) implies that $\omega(T)<\infty$. If $\omega(T)=0$, then $T=T^{\times}, H$ is factorial, and the claim is trivial. Suppose that $\omega(T) \geq 1$. By definition of a prime, we have $\omega(H, p)=1$ for every $p \in P$. Since $H$ is a direct product of $\mathcal{F}(P)$ and $T$, we infer that $\mathcal{A}(H)=P \cup \mathcal{A}(T)$ and

$$
\omega(H)=\sup \{\omega(H, u): u \in \mathcal{A}(H)\}=\sup \{\omega(H, u): u \in \mathcal{A}(T)\}=\sup \{\omega(T, u): u \in \mathcal{A}(T)\}=\omega(T) .
$$

Furthermore, we obtain that $\Delta(H)=\Delta(T), \rho(T)=\rho(H)$, and $\rho_{k}(H)=\rho_{k}(T)$ for all $k \in \mathbb{N}$. Thus all assertions follow from Theorem 3.5 and from Proposition 3.4.

3.1. Cancellative semigroups. In this subsection we gather results on cancellative semigroups implying that the Structure Theorem for Unions holds. They are simple consequences of known results. However, some of them have never been formulated explicitly.

3.1(a) C-monoids, C-domains, and their generalizations. For this kind of domains and monoids $H$, it has been shown that one of the following two conditions holds ([19, Theorem 3.10]):

(a) There exists $k_{0} \in \mathbb{N}$ such that $\rho_{k}(H)=\infty$ for all $k \geq k_{0}$.

(b) For every $k \in \mathbb{N}, \rho_{k}(H)<\infty$ and there is a global bound $M \in \mathbb{N}_{0}$ such that $\rho_{k+1}(H)-\rho_{k}(H) \leq M$. 
Thus $\mathscr{L}(H)$ satisfies the Structure Theorem for Unions by Theorem 2.2. We provide one explicit example of C-domain and refer to [37, 28, 35] for more.

Let $R$ be a Mori domain with complete integral closure $\widehat{R}$. Suppose that $(R: \widehat{R}) \neq\{0\}$, that the class groups $\mathcal{C}_{v}(R)$ and $\mathcal{C}_{v}(\widehat{R})$ are finite, and that the ring $S^{-1} \widehat{R} / S^{-1}(R: \widehat{R})$ is quasi-artinian, where $S$ is the monoid of regular elements of $R$. Then $R$ satisfies one of the above two conditions (see [35, Theorems 6.2 and 7.2], and note that, by Theorem 3.5, the finiteness of the tame degree implies Condition (b)).

3.1(b) Transfer Krull monoids. This class includes all commutative Krull domains, but also maximal orders in central simple algebras. We refer to [20] for a list of examples. Let $H$ be a transfer Krull monoid. Then $H$ has a weak transfer homomorphism $\varphi: H \rightarrow \mathcal{B}\left(G_{0}\right)$, where $G_{0}$ is a subset of an abelian group $G$ and $\mathcal{B}\left(G_{0}\right)$ is the monoid of zero-sum sequences over $G_{0}$. Since $\mathscr{U}_{k}(H)=\mathscr{U}_{k}\left(\mathcal{B}\left(G_{0}\right)\right)$ for all $k \in \mathbb{N}, \mathscr{L}(H)$ satisfies the Structure Theorem for Unions if and only if $\mathscr{L}\left(\mathcal{B}\left(G_{0}\right)\right)$ satisfies the Structure Theorem for Unions. If the Davenport constant $\mathrm{D}\left(G_{0}\right)<\infty$, then $\omega\left(\mathcal{B}\left(G_{0}\right)\right)<\infty$ by [23, Theorem 3.4.10] and hence $\mathscr{L}\left(\mathcal{B}\left(G_{0}\right)\right)$ satisfies the Structure Theorem for Unions by Theorem 3.5 (there are several natural conditions when the finiteness of $\mathrm{D}\left(G_{0}\right)$ and of $\omega\left(\mathcal{B}\left(G_{0}\right)\right)$ are equivalent, see [25, Theorem 4.2]). If $G_{0}=G$, then all sets $\mathscr{U}_{k}(H)$ are intervals (see [21, Theorem 3.1.3] or [18, Theorem 4.1]). Recent progress on the invariants $\rho_{k}(\mathcal{B}(G))$ can be found in [17].

3.2. Semigroups of ideals. In this subsection, all rings and domains are supposed to be commutative. Let $H$ be a cancellative monoid and $r$ an ideal system on $H$. For an ideal $I \in \mathcal{I}_{r}(H)$ we denote by $I^{(k)}$ the $k$-fold product of $I$ with respect to $r$-multiplication. We denote by $\mathcal{I}_{r}^{*}(H) \subseteq \mathcal{I}_{r}(H)$ the subsemigroup of $r$-invertible $r$-ideals. The next lemma deals with strictly $r$-noetherian ideal systems and we recall two examples. First, if $H$ is $s$-noetherian, then $H$ is strictly $s$-noetherian. Second, let $R$ be a noetherian domain, and let $H=R \backslash\{0\}$ be the monoid of its non-zero elements. Then the $d$-system,

$$
d: \mathscr{P}(H) \rightarrow \mathscr{P}(H) \text {, where } \quad X_{d}=\langle X\rangle_{R} \backslash\{0\} \quad \text { for all } \quad X \subseteq H,
$$

consisting of the usual ring ideals, is strictly $d$-noetherian $([32$, Proposition 8.4$])$. We denote by $\mathcal{I}_{d}(R)=$ $\mathcal{I}(R)$ the semigroup of non-zero integral ideals of $R$ (with usual ideal multiplication) and by $\mathcal{I}_{d}^{*}(R)=\mathcal{I}^{*}(R)$ the subsemigroup of invertible ideals.

Lemma 3.7. Let $H$ be a cancellative monoid and let $r$ be a strictly $r$-noetherian ideal system on $H$.

(1) $\mathcal{I}_{r}(H)$ is a BF-monoid.

(2) $\mathcal{I}_{r}^{*}(H)$ is a $v$-noetherian cancellative BF-monoid. In particular, if $R$ is a noetherian domain, then the monoid $\mathcal{I}^{*}(R)$ is a $v$-noetherian cancellative $\mathrm{BF}$-monoid.

Proof. (1) First, we have to show that $\mathcal{I}_{r}(H)$ is unit-cancellative. Let $I, J$ be two non-empty $r$-ideals such that $I \cdot_{r} J=I$. We need to prove that $J=H$. Assume to the contrary that this is not the case. Then Krull's Intersection Theorem [32, Corollary 9.1] implies that $\bigcap_{k \geq 1} J^{(k)}=\varnothing$. But $I=I \cdot r J$ implies that $I=I \cdot{ }_{r} J=I \cdot{ }_{r} J^{(2)}=\ldots=I \cdot_{r} J^{(k)}$ for all $k \in \mathbb{N}$, and hence

$$
I \subseteq \bigcap_{k \geq 1} J^{(k)}=\varnothing,
$$

a contradiction. Thus $\mathcal{I}_{r}(H)$ is a monoid, and it is a BF-monoid by Proposition 3.3(2). 
(2) Clearly, every $r$-invertible $r$-ideal is $r$-cancellative ([23, Section 13.1]), and hence $\mathcal{I}_{r}^{*}(H)$ is a cancellative monoid. The remaining statements follow from [24, Example 2.1].

Lemma 3.7 shows that semigroups of ( $r$-invertible) $r$-ideals are BF-monoids which often satisfy additional noetherian properties. The verification of the assumptions needed to enforce the validity of the Structure Theorem for Unions clearly depends on the ideal system $r$ and the class of monoids and domains under consideration. Much is already known for $r$-invertible $r$-ideals which are cancellative. For example, if $R$ is a $v$-noetherian weakly Krull domain with non-zero conductor, then $\mathscr{L}\left(\mathcal{I}_{v}^{*}(R)\right)$ satisfies the Structure Theorem for Unions (use [23, Theorem 3.7.1] and Subsection 3.1). To provide an explicit example of not necessarily cancellative semigroups of ideals, we consider one-dimensional noetherian domains. Note that by an irreducible ideal we mean an ideal which is an irreducible element in the semigroup of ideals under consideration.

Corollary 3.8. Let $R$ be a one-dimensional noetherian domain such that its integral closure $\bar{R}$ is a finitely generated $R$-module, and let $\mathfrak{f}=(R: \bar{R})$ and $\mathcal{P}^{*}=\{\mathfrak{p} \in \mathfrak{X}(R): \mathfrak{p} \supseteq \mathfrak{f}\}$. If the following condition:

(C) For every $\mathfrak{p} \in \mathcal{P}^{*}$, there are only finitely many irreducible primary ideals $\mathfrak{q}$ with $\sqrt{\mathfrak{q}}=\mathfrak{p}$,

holds, then $\mathscr{L}(\mathcal{I}(R))$ satisfies the Structure Theorem for Unions. In particular:

(1) If $R$ is a Cohen-Kaplansky domain, then Condition (C) holds.

(2) If $R$ is an order in a quadratic number field $K$ with discriminant $d_{K}$ and conductor $\mathfrak{f}=f \mathbb{Z}$, then Condition (C) is equivalent to each of the following:

(C1) For every $\mathfrak{p} \in \mathcal{P}^{*}$, there are only finitely many invertible irreducible primary ideals $\mathfrak{q}$ with $\sqrt{\mathfrak{q}}=\mathfrak{p}$.

(C2) For every $\mathfrak{p} \in \mathcal{P}^{*}$, there is precisely one prime ideal of $\bar{R}$ lying above $\mathfrak{p}$.

(C3) For every prime divisor $p$ of $f$ the Legendre symbol $\left(\frac{d_{K}}{p}\right) \neq-1$.

Proof. Let $\pi: \mathfrak{X}(\bar{R}) \rightarrow \mathfrak{X}(R)$ be defined by $\pi(\mathfrak{P})=\mathfrak{P} \cap R$. By the Primary Decomposition Theorem, every ideal $I \in \mathcal{I}(R)$ is, up to order, a unique product of primary ideals having pairwise distinct radicals. The set $\mathcal{P}=\mathfrak{X}(R) \backslash \mathcal{P}^{*}$ is the set of prime elements of $\mathcal{I}(R)$, and every ideal $I \in \mathcal{I}(R)$ can be written uniquely as a product of powers of prime elements and of primary elements with radicals in $\mathcal{P}^{*}$. Therefore $\mathcal{I}(R)=\mathcal{F}(\mathcal{P}) \times T$ where $T \subseteq \mathcal{I}(R)$ is the submonoid generated by atoms which are not prime. Condition (C) states that the monoid $T$ is finitely generated. Thus the assumptions of Theorem 3.6 hold, and $\mathscr{L}(\mathcal{I}(R))$ satisfies the Structure Theorem for Unions.

It remains to discuss the two special cases.

(1) By definition, a domain is a Cohen-Kaplansky domain if it is atomic and has only finitely many non-associated atoms. Suppose $R$ is a Cohen-Kaplansky domain. Then $R$ is a one-dimensional, semilocal noetherian domain, $\bar{R}$ is a finitely generated $R$-module, and both ideal semigroups $\mathcal{I}(R)$ and $\mathcal{I}^{*}(R)$ are finitely generated by [1, Theorem 4.3]. Thus Condition (C) holds. Note that Cohen-Kaplansky domains are precisely the domains for which $\mathcal{I}(R)$ is finitely generated.

(2) Suppose that $R$ is an order in a quadratic number field. The equivalence of the given conditions is classical and can be found in [27, p. 36] and [33, Theorem 5.8.8]. Clearly, (C2) states that $\pi: \mathfrak{X}(\bar{R}) \rightarrow \mathfrak{X}(R)$ is bijective. 
3.3. Semigroups of modules. The study of direct-sum decomposition of modules is a classical topic of module theory. There is an overwhelming literature investigating for which classes of modules the KrullRemak-Schmidt-Azumaya Theorem (stating that direct-sum decomposition is unique) holds, or how badly it fails.

The last decade has seen a new semigroup-theoretical approach to the study of direct-sum decomposition. To highlight this approach, we need some notation. Let $R$ be a (not necessarily commutative) ring and $\mathcal{C}$ a class of (left) $R$-modules which is closed under finite direct sums, direct summands, and isomorphisms. This means that whenever $M, M_{1}$ and $M_{2}$ are $R$-modules with $M \cong M_{1} \oplus M_{2}$, we have $M \in \mathcal{C}$ if and only if $M_{1}, M_{2} \in \mathcal{C}$. Let $\mathcal{V}(\mathcal{C})$ denote a set of representatives of isomorphism classes in $\mathcal{C}$, and for a module $M$ in $\mathcal{C}$ let $[M]$ denote the unique element in $\mathcal{V}(\mathcal{C})$ isomorphic to $M$. Then $\mathcal{V}(\mathcal{C})$ is an (additive) reduced commutative semigroup, where the operation is defined by $[M]+[N]=[M \oplus N]$, the identity is the zero-module, and the irreducible elements are the isomorphism classes of indecomposable modules. Of course, the semigroup $\mathcal{V}(\mathcal{C})$ carries all the information about the direct-sum behavior of modules in $\mathcal{C}$.

If $\operatorname{End}_{R}(M)$ is semilocal for each $M \in \mathcal{C}$, then $\mathcal{V}(\mathcal{C})$ is a Krull monoid by a result of Facchini [11, Theorem 3.4]. In this case $\mathscr{L}(\mathcal{V}(\mathcal{C}))$ can be studied with the theory of Krull monoids and we refer back to Subsection 3.1. This pioneering result was the starting point pushing a new strategy to understand the direct-sum behavior of modules by studying the algebraic and arithmetic properties of $\mathcal{V}(\mathcal{C})$ (see $[40,14,3]$ for surveys and [2, p. 315] for a detailed program description; in [10] $\omega$-invariants of semigroup of modules are studied in terms of a so-called semi-exchange property).

To begin, direct-sum decompositions can be as wild as factorizations in arbitrary commutative semigroups. Indeed, we know from a result of Facchini and Wiegand [15, Theorem 2.1] that every reduced Krull monoid $H$ is isomorphic to a monoid of modules $\mathcal{V}(\mathcal{C})$, where $\mathcal{C}$ is a class of finitely generated projective modules with semilocal endomorphism rings. And by results of Bergman and Dicks, for every reduced commutative semigroup $H$ (with order-unit) there exists a class $\mathcal{C}$ of finitely generated projective modules over a right and left hereditary $k$-algebra $R$ such that $\mathcal{V}(\mathcal{C})$ is isomorphic to $H$ ([12, Corollary 5]).

So far, the focus of research has been on classes $\mathcal{C}$ of modules for which $\mathcal{V}(\mathcal{C})$ is cancellative, but other classes of semigroups appear naturally (see the Seven-Step hierarchy given by Facchini in [13]). In order to apply Theorem 3.6 to a monoid of modules $\mathcal{V}(\mathcal{C})$, we analyze its assumptions in the present setting. Clearly, $\mathcal{V}(\mathcal{C})$ being finitely generated means that, up to isomorphism, there are only finitely many indecomposable $R$-modules, and unit-cancellativity reads as follows:

$$
\text { If } M, N \text { are modules from } \mathcal{C} \text { such that } \quad M \cong M \oplus N, \text { then } \quad N=0 .
$$

Thus unit-cancellativity states that all modules have to be directly finite (or Dedekind finite). Clearly, finitely generated modules over commutative rings are directly finite. Furthermore, modules with finite uniform dimension are directly finite, and so are finitely generated projective modules over unit-regular rings. For more information on directly finite modules we refer to [30, 34, 6].

We summarize our discussion in the following corollary.

Corollary 3.9. Let $R$ be a ring and $\mathcal{C}$ be a class of $R$-modules which is closed under finite direct sums, direct summands, and isomorphisms. Suppose that all modules in $\mathcal{C}$ are directly finite and that there are, 
up to isomorphism, only finitely many indecomposable modules in $\mathcal{C}$. Then $\mathscr{L}(\mathcal{V}(\mathcal{C}))$ satisfies the Structure Theorem for Unions.

Proof. This follows immediately from Theorem 3.6.

3.4. Power monoids. Given an additively written commutative semigroup $H$, the power semigroup $\mathscr{P}_{\text {fin }}(H)$ is the semigroup of all finite non-empty subsets of $H$ endowed with the operation of set addition. The study of sumsets is in the center of interests in arithmetic combinatorics [31], but the abstract semigroup theoretical point of view was adopted only recently in [16].

In general, $\mathscr{P}_{\text {fin }}(H)$ need not be unit-cancellative. If, for instance, $H$ contains a finite non-trivial subgroup $G$, then $G \in \mathscr{P}_{\text {fin }}(H)$ and $G+G=G$, but $G$ is not a unit in $\mathscr{P}_{\text {fin }}(H)$. This shows that a necessary condition for $\mathscr{P}_{\text {fin }}(H)$ to be a monoid is that $H$ is torsion-free, and for a commutative, cancellative semigroup it is well known that torsion-freeness is equivalent to being linearly orderable. We recall that a semigroup $H$ is linearly orderable if there exists a total order $\preceq$ on the set $H$ such that $x+z \prec y+z$ for all $x, y, z \in H$ with $x \prec y$ (note that linearly orderable semigroups are cancellative).

In general, we cannot expect arithmetical finiteness properties from $\mathscr{P}_{\text {fin }}(H)$ (to provide an example, if $H=\left(\mathbb{N}_{0},+\right)$, then the set of distances of $\mathscr{P}_{\text {fin }}(H)$ is equal to $\mathbb{N}$ by [16]). However, finitely generated subsemigroups of $\mathscr{P}_{\text {fin }}(H)$ do satisfy arithmetical finiteness properties. To see this, consider a subset $\mathcal{E} \subseteq \mathscr{P}_{\text {fin }}(H)$. We denote by $H(\mathcal{E})$ the subsemigroup of $\mathscr{P}_{\text {fin }}(H)$ generated by $\mathcal{E}$. Clearly, if $\mathcal{E}$ is a family of singletons, then $H(\mathcal{E})$ is isomorphic to the subsemigroup of $H$ generated by the elements occurring in the singletons of $\mathcal{E}$. Moreover, we have the following result.

Corollary 3.10. Let $H$ be a reduced linearly orderable $\mathrm{BF}$-monoid, and let $\mathcal{E} \subseteq \mathcal{P}_{\text {fin }}(H)$ be a finite subset. Then $H(\mathcal{E})$ is a finitely generated $\mathrm{BF}$-monoid and $\mathscr{L}(H(\mathcal{E}))$ satisfies the Structure Theorem for Unions.

Proof. It follows from [16] that $H(\mathcal{E})$ is a finitely generated BF-monoid, and hence Theorem 3.6 implies the validity of the Structure Theorem for Unions.

The special case of Corollary 3.10, when $H$ is the monoid of non-negative integers under addition, lies at the heart of the following remark, in which we highlight a few essential differences between the classical cancellative setting and the broader setting considered in the present work.

Remarks 3.11. Let $H$ be an atomic monoid.

(1) By [32, Theorem 3.6], every reduced finitely generated commutative semigroup is $s$-noetherian. However, in contrast to Proposition 3.1 it need not be atomic (see [38, Theorem 3 and Example 6]).

(2) If $H$ is cancellative and $a \in H$ with $|\mathrm{Z}(a)| \geq 2$, then $2+\sup \Delta(\mathrm{L}(a)) \leq \mathrm{c}(a)$ by [23, Lemma 1.6.2], but in the more general setting of Proposition 3.2, the inequality $1+\sup \Delta(\mathrm{L}(a)) \leq \mathrm{c}(a)$ can be sharp.

(3) Let $\sim_{H}=\{(x, y) \in \mathbf{Z}(H) \times \mathbf{Z}(H): \pi(x)=\pi(y)\}$ be the monoid of relations of $H$, and assume first that $H$ is cancellative. Then $\sim_{H}$ is a fundamental tool for studying the arithmetic of $H$. In particular, it is easy to see that the canonical embedding $j: \sim_{H} \hookrightarrow \mathrm{Z}(H) \times \mathrm{Z}(H)$ is a divisor homomorphism. Thus $\sim_{H}$ is a Krull monoid, and it is finitely generated if so is $H$.

However, if $H$ is not cancellative, things are quite different. Indeed, suppose that $H$ is reduced and there exist $a, b, c \in H$ with $b \neq c$ and $a b=a c$. Then none of $a, b$, and $c$ is a unit, and hence none of $Z(a)$, 
$\mathbf{Z}(b)$, and $\mathbf{Z}(c)$ is empty. Let $z \in \mathbf{Z}(a b), z_{a} \in \mathbf{Z}(a), z_{b} \in \mathbf{Z}(b)$, and $z_{c} \in \mathbf{Z}(c)$. Then $(z, z)$ and $\left(z_{a}, z_{a}\right)$ both belong to $\sim_{H}$, and $(z, z)=\left(z_{a}, z_{a}\right)\left(z_{b}, z_{c}\right)$ in $\mathbf{Z}(H) \times \mathbf{Z}(H)$. Yet, $\left(z_{b}, z_{c}\right)$ is not in $\sim_{H}$, and it follows that $j$ is not a divisor homomorphism.

Now, for a fixed integer $n \geq 2$ let $H$ be the submonoid of $\mathscr{P}_{\text {fin }}\left(\mathbb{N}_{0}\right)$ generated by the sets $\llbracket 0,1 \rrbracket$ and $A=\{1\} \cup(2 \cdot \llbracket 0, n \rrbracket)$. It is seen that $h \llbracket 0,1 \rrbracket+k A$ is an interval for all $h \in \mathbb{N}$ and $k \in \mathbb{N}_{0}$. Therefore, we find that $\llbracket 0,1 \rrbracket$ is prime in $H$ and $\mathcal{A}(H)=\{\llbracket 0,1 \rrbracket, A\}$. In particular, $H$ is a finitely generated, reduced, atomic monoid. However, we claim that $\sim_{H}$ is not finitely generated.

In fact, we will show that $(\llbracket 0,1 \rrbracket+k A,(2 n k+1) \llbracket 0,1 \rrbracket)$ is an atom of $\sim_{H}$ for every $k \in \mathbb{N}_{0}$. (Since $H$ is written additively, so will be its factorization monoid $\mathbf{Z}(H)$.) Suppose for a contradiction that this is not true. Then there must exist $k \in \mathbb{N}_{0}, h \in \llbracket 0, k \rrbracket$, and $\ell \in \llbracket 0,2 n k+1 \rrbracket$ with $h+\ell \geq 1$ such that

$$
(\llbracket 0,1 \rrbracket+k A,(2 n k+1) \llbracket 0,1 \rrbracket)=(h A, \ell \llbracket 0,1 \rrbracket)(\llbracket 0,1 \rrbracket+(k-h) A,(2 n k+1-\ell) \llbracket 0,1 \rrbracket) \quad \text { in } \quad \sim_{H} .
$$

But this implies that $h A=\llbracket 0, \ell \rrbracket$, which is impossible, since $h A$ is not an interval for $h \neq 0$, and on the other hand, $\{0\}=0 A=\llbracket 0, \ell \rrbracket$ only for $\ell=0$ (recall that we are requiring $h+\ell \geq 1$ ).

(4) Let $P$ be a maximal set of pairwise non-associated prime elements in $H$, and let $T$ be the set of all $a \in H$ for which there does not exist any $p \in P$ such that $p \mid a$. Of course, $T$ is a submonoid of $H$, and by Lemma 3.1 we have $H=\mathcal{F}(P) T$. If $H$ is cancellative, then $H$ is isomorphic to the direct product $\mathcal{F}(P) \times T$. However, this need not be the case if $H$ is not cancellative.

Indeed, assume as in point (3) that $H$ is the submonoid of $\mathscr{P}_{\text {fin }}\left(\mathbb{N}_{0}\right)$ generated by $\llbracket 0,1 \rrbracket$ and $A=$ $\{1\} \cup(2 \cdot \llbracket 0, n \rrbracket)$, where $n$ is a fixed integer $\geq 2$. We have already noted that $H$ is reduced, and $\llbracket 0,1 \rrbracket$ is prime and $A$ is irreducible in $H$. So we necessarily have $P=\{\llbracket 0,1 \rrbracket\}$ and $T=\left\{k A: k \in \mathbb{N}_{0}\right\}$, and $H$ is not isomorphic to $\mathcal{F}(P) \times T$, because $\llbracket 0,1 \rrbracket+A=(2 n+1) \llbracket 0,1 \rrbracket$ in $H$.

(5) It is known that if $H$ is finitely generated and cancellative, then $H$ has accepted elasticity ([23, Theorem 3.1.4]). Yet, this need not be true in the non-cancellative setting.

To see why, let $H$ be given as in the second paragraph of point (4), and let $X$ be a non-unit element of $H$. Then $X=h \llbracket 0,1 \rrbracket+l A$ for some $h, l \in \mathbb{N}_{0}$ with $h+l \geq 1$. We seek an explicit description of the set of lengths, $\mathrm{L}(X)$, of $X$. To start with, note that if $h=0$, then $\mathrm{L}(X)=\{l\}$, since $l A \neq j A$ for all $j \in \mathbb{N}_{0}$ with $j \neq l$ and, on the other hand, $\llbracket 0,1 \rrbracket$ cannot be a divisor of $l A$ (in $H$ ), otherwise $l A$ would be an interval. So assume $h \geq 1$, and let $h=2 n q+r$, where $q \in \mathbb{N}_{0}, r \in \llbracket 0,2 n-1 \rrbracket$, and $q+r \geq 1$. Then $X=\llbracket 0,2 n(q+l)+r \rrbracket$, and we look for all pairs $(j, m)$ of non-negative integers such that

$$
\llbracket 0,2 n(q+l)+r \rrbracket=j \llbracket 0,1 \rrbracket+m A .
$$

This condition implies that $2 n(q+l)+r=j+m \max A=j+2 m n$, and is actually possible if and only if

$$
j=2 n(q+l-m)+r \quad \text { for some } m \in \llbracket 0, q+l-\varepsilon_{r} \rrbracket,
$$

where $\varepsilon_{r}=1$ if $r=0$ and $\varepsilon_{r}=0$ otherwise. It follows that

$$
\mathrm{L}(X)=\left\{(2 n-1) x+q+l+r: x \in \llbracket \varepsilon_{r}, q+l \rrbracket\right\},
$$

whence $\min \mathrm{L}(X)=q+l+r+(2 n-1) \varepsilon_{r}, \max \mathrm{L}(X)=2 n(q+l)+r$, and

$$
\rho(X)=\frac{2 n(q+l)+r}{q+l+r+(2 n-1) \varepsilon_{r}}<2 n=\lim _{q+l \rightarrow \infty} \frac{2 n(q+l)+r}{q+l+r+(2 n-1) \varepsilon_{r}},
$$

where the limit on the right-most side is uniform with respect to the parameter $r$. 
Therefore, we conclude that $\rho(H)=2 n \neq \rho(X)$ for every $X \in H$. In particular, $H$ does not have accepted elasticity, although it is finitely generated (in fact, 2-generated).

With this in hand, we observe that, by Proposition 3.4(1), $\rho_{k}(H)<\infty$ for all $k \in \mathbb{N}$. We want to show that $\rho_{k+1}(H)-\rho_{k}(H) \leq 2 n$ for all sufficiently large $k \in \mathbb{N}$.

Indeed, let $k$ be an integer $\geq 2 n$, and write $k=(2 n-1) t+s$, where $t \in \mathbb{N}$ and $s \in \llbracket 0,2 n-2 \rrbracket$. Our goal is to compute $\rho_{k}$. To this end, we seek all triples $(q, l, r) \in \mathbb{N}_{0}^{3}$ with $q+r \geq 1$ and $0 \leq r<2 n$ such that $k \in \mathrm{L}(\llbracket 0,2 n(q+l)+r \rrbracket)$, which is equivalent by $(3.2)$ to having that

$$
k=(2 n-1) x+q+l+r \quad \text { for some } \quad x \in \llbracket \varepsilon_{r}, q+l \rrbracket .
$$

Since we have already seen that $\max \mathrm{L}(\llbracket 0,2 n(q+l)+r \rrbracket)=2 n(q+l)+r$, we can just look for the set $\mathcal{M}$ of those triples $(q, l, r) \in \mathbb{N}_{0}^{3}$ with $q+r \geq 1$ and $0 \leq r<2 n$ for which Condition (3.3) is fulfilled and $2 n(q+l)+r$ is maximal (note that $\mathcal{M}$ is non-empty).

Let $(q, l, r) \in \mathcal{M}$. Then (3.3) is necessarily satisfied with $x=\varepsilon_{r}$. Otherwise, taking $x^{\prime}=x-1$ and $q^{\prime}=q+2 n-1$ would give that $\left(q^{\prime}, l, r\right) \in \mathbb{N}_{0}^{3}, q^{\prime}+r \geq 1$, and $x^{\prime} \in \llbracket \varepsilon, q^{\prime}+l \rrbracket$, but also

$$
2 n\left(q^{\prime}+l\right)+r>2 n(q+l)+r \quad \text { and } \quad k=(2 n-1) x^{\prime}+q^{\prime}+l+r,
$$

in contradiction to the maximality of the triple $(q, l, r)$. This shows that $\mathcal{M}$ is the set of all triples $(q, l, r) \in \mathbb{N}_{0}^{3}$ with $q+r \geq 1$ and $0 \leq r<n$ for which $2 n(q+l)+r$ is maximal and

$$
q+l=(2 n-1)\left(t-\varepsilon_{r}\right)+s-r .
$$

Now we notice that $(2 n-1)\left(t-\varepsilon_{r}\right)+s-r \geq 1$ for every $r \in \llbracket 0,2 n-1 \rrbracket$, since having assumed $k \geq 2 n$ implies that $t \geq 1$, and that $s \geq 1$ for $t=1$. This means that, however we choose an $r \in \llbracket 0,2 n-1 \rrbracket$, there are $q, l \in \mathbb{N}_{0}$ with $q+r \geq 1$ such that (3.4) is satisfied, which ultimately yields

$$
\rho_{k}(H)=\max \left\{(2 n-1)\left(t-\varepsilon_{r}\right)+s-r: r \in \llbracket 0, n-1 \rrbracket\right\}=2 n(k-1)+1 .
$$

Putting it all together, we thus conclude that $\rho_{k+1}(H)-\rho_{k}(H)=2 n$ for every integer $k \geq 2 n$.

Incidentally, it seems worth noting that $\omega(H)=2 n+1$ : This comes a consequence of what we have already observed in points (3) and (4), namely the fact that $\llbracket 0,1 \rrbracket$ is prime and $A$ divides $k \llbracket 0,1 \rrbracket$ for $k \geq 2 n+1$. In particular, we obtain from here and the above that $\rho_{k+1}(H)-\rho_{k}(H) \leq \max (1, \omega(H)-1)$ for all sufficiently large $k \in \mathbb{N}$, which is consistent with the bound provided in the cancellative setting by Theorem 3.5 and leads us to ask whether the same inequality is actually true for any finitely generated monoid (if not for every monoid).

(6) If $H$ is as in Theorem 3.5, then the assumptions made in Theorem 2.2(2) need not hold, not even in the cancellative case. To be more precise, let $H$ be a cancellative finitely generated monoid. Then $\rho(H)<\infty$ and there is an $L^{*} \in \mathscr{L}(H)$ such that $\rho\left(L^{*}\right)=\rho(H)$ (see [23, Theorem 3.1.4]). However, there need not be an $L^{*} \in \mathscr{L}(H)$ which is an AP with difference min $\Delta(H)$ and satisfies $\rho\left(L^{*}\right)=\rho(H)$ (see [18, Example 3.1]). For numerical monoids, sufficient conditions implying the existence of such an $L^{*} \in \mathscr{L}(H)$ are given in [5, Theorem 6.6]. 


\section{A semigroup not satisfying the Structure Theorem for Unions}

In this section we provide the first example of a semigroup whose system of sets of lengths does not satisfy the Structure Theorem for Unions. Our example is, actually, a locally tame Krull monoid with finite set of distances. Recall that globally tame cancellative monoids satisfy the Structure Theorem for Unions (Theorem 3.5), as well as the Structure Theorem for Sets of Lengths ([25, Theorem 5.1]).

Proposition 4.1. Let $L \subseteq \mathbb{N}_{\geq 2}$ be a finite subset such that $|L| \geq 2$. Then there exists a finitely generated reduced Krull monoid $H$ such that $L \in \mathscr{L}(H)$ and the following properties hold:

(i) $\max \Delta(H)=\max \Delta(L)$.

(ii) $\mathscr{U}_{\min L}(H)=L$.

(iii) $\rho_{\ell \min L+\nu}(H)=\ell \max L+\nu$ for all $\nu \in \llbracket 0$, $\min L-1 \rrbracket$ and $\ell \in \mathbb{N}_{0}$.

Comment. Since $L \in \mathscr{L}(H)$, it is clear that $\Delta(L) \subseteq \Delta(H)$. Yet, we cannot expect equality in general, as we know from Proposition 2.9 that $\min \Delta(H)=\operatorname{gcd} \Delta(H)$, which does not necessarily hold for $\Delta(L)$.

Proof. Set $L=\left\{m_{1}, m_{2}+1, \ldots, m_{s}+1\right\} \subseteq \mathbb{N}_{\geq 2}$, where $s=|L| \geq 2, m_{1} \leq m_{2}$, and $m_{i}<m_{i+1}$ for every $i \in \llbracket 2, s-1 \rrbracket$. Take $P=\left\{u_{i, j}: i \in \llbracket 1, s \rrbracket\right.$ and $\left.j \in \llbracket 1, m_{i} \rrbracket\right\}$ to be a finite set with $|P|=m_{1}+\ldots+m_{s}$, and let $F=\mathcal{F}(P)$ be the free abelian monoid with basis $P$. For each $i \in \llbracket 2, s \rrbracket$ we set

$$
u_{i, 0}=u_{1,1} \cdot \ldots \cdot u_{1, m_{1}}\left(u_{i, 1} \cdot \ldots \cdot u_{i, m_{i}}\right)^{-1} \in \mathrm{q}(F)
$$

and we define $H=[\mathcal{A}] \subseteq \mathrm{q}(F)$, where

$$
\mathcal{A}=\left\{u_{i, j}: i \in \llbracket 1, s \rrbracket \text { and } j \in \llbracket 1, m_{i} \rrbracket\right\} \cup\left\{u_{i, 0}: i \in \llbracket 2, s \rrbracket\right\} .
$$

We note that $\mathrm{q}(H)=\mathrm{q}(F)$, and we proceed in a series of steps.

1. Description of $H$. To start with, we seek a more explicit description of the monoid $H$. For this, note first that, if $\alpha_{i, j} \in \mathbb{Z}$ for $i \in \llbracket 1, s \rrbracket, j \in \llbracket 1, m_{i} \rrbracket$ and $\beta_{i} \in \mathbb{Z}$ for $i \in \llbracket 2, s \rrbracket$, then we have by (4.1) that

$$
\prod_{i=1}^{s} \prod_{j=1}^{m_{i}} u_{i, j}^{\alpha_{i, j}} \prod_{i=2}^{s} u_{i, 0}^{\beta_{i}}=\prod_{j=1}^{m_{1}} u_{1, j}^{\alpha_{1, j}+\sum_{i=2}^{s} \beta_{i}} \prod_{i=2}^{s} \prod_{j=1}^{m_{i}} u_{i, j}^{\alpha_{i, j}-\beta_{i}} .
$$

Fix $x \in \mathrm{q}(F)$. Then there are uniquely determined $\gamma_{i, j}(x) \in \mathbb{Z}$, for $i \in \llbracket 1, s \rrbracket$ and $j \in \llbracket 1, m_{i} \rrbracket$, such that

$$
x=\prod_{i=1}^{s} \prod_{j=1}^{m_{i}} u_{i, j}^{\gamma_{i, j}(x)} .
$$

Now, for each $i \in \llbracket 1, s \rrbracket$ we define

$$
\gamma_{i}(x)=\min \left\{\gamma_{i, 1}(x), \ldots, \gamma_{i, m_{i}}(x)\right\} \quad \text { and } \quad \gamma_{i}^{\prime}(x)=\max \left\{0,-\gamma_{i}(x)\right\} .
$$

Furthermore, we denote by $\mathbf{Z}_{\mathcal{A}}(x)$ the set of all tuples $\left(\left(\alpha_{i, j}\right)_{1 \leq i \leq s, 1 \leq j \leq m_{i}}, \beta_{2}, \ldots, \beta_{s}\right) \in \mathbb{N}_{0}^{m_{1}+\ldots+m_{s}+s-1}$ such that

$$
x=\prod_{i=1}^{s} \prod_{j=1}^{m_{i}} u_{i, j}^{\alpha_{i, j}} \prod_{i=2}^{s} u_{i, 0}^{\beta_{i}} .
$$

Lastly, for every $\alpha=\left(\left(\alpha_{i, j}\right)_{1 \leq i \leq s, 1 \leq j \leq m_{i}}, \beta_{2}, \ldots, \beta_{s}\right) \in \mathbb{N}_{0}^{m_{1}+\ldots+m_{s}+s-1}$ we let

$$
|\alpha|=\sum_{i=1}^{s} \sum_{j=1}^{m_{i}} \alpha_{i, j}+\sum_{i=2}^{s} \beta_{i}
$$


be the length of $\alpha$, and we set

$$
\mathrm{L}_{\mathcal{A}}(x)=\left\{|\alpha|: \alpha \in \mathrm{Z}_{\mathcal{A}}(x)\right\} .
$$

By definition, $x \in H$ if and only if $\mathbf{Z}_{\mathcal{A}}(x) \neq \varnothing$. Our goal is to give a simpler description of $\mathbf{Z}_{\mathcal{A}}(x)$. To this end, let $B(x)$ be the set of all tuples $\left(\beta_{2}, \ldots, \beta_{s}\right) \in \mathbb{N}_{0}^{s-1}$ such that

$$
\sum_{i=2}^{s} \beta_{i} \leq \gamma_{1}(x) \quad \text { and } \quad \beta_{i} \geq-\gamma_{i}(x) \text { for each } i \in \llbracket 2, s \rrbracket .
$$

It is clear that $B(x) \neq \varnothing$ if and only if $\gamma_{1}(x) \geq \sum_{i=2}^{s} \gamma^{\prime}(x)$, and we will prove that $B(x) \neq \varnothing$ if and only if $Z_{\mathcal{A}}(x) \neq \varnothing$, whence we can conclude that

$$
H=\left\{x \in \mathrm{q}(F): \gamma_{1}(x) \geq \sum_{i=2}^{s} \gamma_{i}^{\prime}(x)\right\} .
$$

To begin, let $\beta=\left(\beta_{2}, \ldots, \beta_{s}\right) \in B(x)$, and for every $j \in \llbracket 1, m_{1} \rrbracket$ define $\alpha_{1, j}=\gamma_{1, j}(x)-\sum_{i=2}^{s} \beta_{i}$. Then

$$
\alpha_{1, j} \geq \gamma_{1}(x)-\sum_{i=2}^{s} \beta_{i} \geq 0
$$

Similarly, for all $i \in \llbracket 2, s \rrbracket$ and $j \in \llbracket 1, m_{i} \rrbracket$ set $\alpha_{i, j}=\gamma_{i, j}(x)+\beta_{i}$. Again, we have $\alpha_{i, j} \geq \gamma_{i}(x)+\beta_{i} \geq 0$. It follows by (4.2) and (4.3) that

$$
\mathbf{Z}_{x}(\beta):=\left(\left(\alpha_{i, j}\right)_{1 \leq i \leq s, 1 \leq j \leq m_{i}}, \beta\right) \in \mathbf{Z}_{\mathcal{A}}(x) .
$$

Consider the map $\mathbf{Z}_{x}: B(x) \rightarrow \mathbf{Z}_{\mathcal{A}}(x), \beta \mapsto \mathbf{Z}_{x}(\beta)$. This is obviously injective, and we want to show that it is also surjective. Indeed, let $\alpha=\left(\left(\alpha_{i, j}\right)_{1 \leq i \leq s, 1 \leq j \leq m_{i}}, \beta_{2}, \ldots, \beta_{s}\right) \in \mathbf{Z}_{\mathcal{A}}(x)$. By (4.2) and (4.3), we have

$$
\alpha_{1, j}=\gamma_{1, j}(x)-\sum_{i=2}^{s} \beta_{i} \text { for each } j \in \llbracket 1, m_{1} \rrbracket
$$

and

$$
\alpha_{i, j}=\gamma_{i, j}(x)+\beta_{i} \text { for all } i \in \llbracket 2, s \rrbracket \text { and } j \in \llbracket 1, m_{i} \rrbracket .
$$

So, since $\alpha_{i, j} \geq 0$ for every $i \in \llbracket 1, s \rrbracket$ and $j \in \llbracket 1, m_{i} \rrbracket$, we get $\beta=\left(\beta_{2}, \ldots, \beta_{s}\right) \in B(x)$, and this entails (by construction) that $\mathbf{Z}_{x}(\beta)=\alpha$. Therefore, we can conclude that the map $\mathbf{Z}_{x}: B(x) \rightarrow \mathbf{Z}_{\mathcal{A}}(x)$ is bijective, which, in turn, implies that $B(x)$ is non-empty if and only if so is $\mathbf{Z}_{\mathcal{A}}(x)$, as was desired. In addition,

$$
\mathbf{L}_{\mathcal{A}}(x)=\left\{\left|\mathbf{Z}_{x}(\beta)\right|: \beta \in B(x)\right\} .
$$

2. Claim: $H$ is a reduced, finitely generated Krull monoid.

Since $\gamma_{i}(1)=0$ for all $i \in \llbracket 1, s \rrbracket$, we find $B(1)=\{(0, \ldots, 0)\}$. Thus, we also have $\mathbf{Z}_{\mathcal{A}}(1)=\{(0, \ldots, 0)\}$. Now let $x \in H^{\times}$, and choose $z \in \mathbf{Z}_{\mathcal{A}}(x)$ and $w \in \mathbf{Z}_{\mathcal{A}}\left(x^{-1}\right)$. Then $z+w \in \mathbf{Z}_{\mathcal{A}}(1)=\{(0, \ldots, 0)\}$, and hence $0=|z+w|=|z|+|w|$. This is possible only if $z=(0, \ldots, 0)$, and it shows that $x=1$.

On the other hand, $H$ is finitely generated (by definition). Hence, $H$ is Krull if and only if it is root closed (see [23, Theorem 2.7.14]). Accordingly, let $x \in \mathrm{q}(H)=\mathrm{q}(F)$ and $t \in \mathbb{N}$ such that $x^{t} \in H$. Since $\gamma_{1}\left(x^{t}\right)=t \gamma_{1}(x)$ and $\gamma_{i}^{\prime}\left(x^{t}\right)=t \gamma_{i}^{\prime}(x)$, it follows by (4.4) that $x \in H$, and we are done.

3. Sets of lengths. Let $x \in H$. We set

$$
C(x)=\sum_{i=1}^{s} \sum_{j=1}^{m_{i}} \gamma_{i, j}(x)+\sum_{i=2}^{s}\left(-m_{1}+m_{i}+1\right) \gamma_{i}^{\prime}(x) \quad \text { and } \quad k(x)=\gamma_{1}(x)-\sum_{i=2}^{s} \gamma_{i}^{\prime}(x) .
$$


We get from (4.4) that $k(x) \in \mathbb{N}_{0}$. We claim that

$$
\mathrm{L}_{\mathcal{A}}(x)=C(x)+\left\{\sum_{i=2}^{s}\left(-m_{1}+m_{i}+1\right) \beta_{i}: \beta_{2}, \ldots, \beta_{s} \in \mathbb{N}_{0} \text { and } \sum_{i=2}^{s} \beta_{i} \leq k(x)\right\} .
$$

Indeed, we have by the above discussion (see, in particular, Step 1) that

$$
\begin{aligned}
B(x) & =\left\{\left(\beta_{2}, \ldots, \beta_{s}\right) \in \mathbb{N}_{0}^{s-1}: \beta_{2} \geq \gamma_{2}^{\prime}(x), \ldots, \beta_{s} \geq \gamma_{s}^{\prime}(x), \text { and } \sum_{i=2}^{s} \beta_{i} \leq \gamma_{1}(x)\right\} \\
& =\left(\gamma_{2}^{\prime}(x), \ldots, \gamma_{s}^{\prime}(x)\right)+\left\{\left(\beta_{2}, \ldots, \beta_{s}\right) \in \mathbb{N}_{0}^{s-1}: \sum_{i=2}^{s} \beta_{i} \leq k(x)\right\} .
\end{aligned}
$$

Then, let $\left(\beta_{2}, \ldots, \beta_{s}\right) \in \mathbb{N}_{0}^{s-1}$ be such that $\sum_{i=2}^{s} \beta_{i} \leq k(x)$, and set $\alpha=Z_{x}\left(\gamma_{2}^{\prime}(x)+\beta_{2}, \ldots, \gamma_{s}^{\prime}(x)+\beta_{s}\right)$. Plugging in the definitions, we obtain

$$
\begin{aligned}
|\alpha| & =\sum_{j=1}^{m_{1}}\left(\gamma_{1, j}(x)-\sum_{i=2}^{s}\left(\gamma_{i}^{\prime}(x)+\beta_{i}\right)\right)+\sum_{i=2}^{s} \sum_{j=1}^{m_{i}}\left(\gamma_{i, j}(x)+\gamma_{i}^{\prime}(x)+\beta_{i}\right)+\sum_{i=2}^{s}\left(\gamma_{i}^{\prime}(x)+\beta_{i}\right) \\
& =\sum_{i=1}^{s} \sum_{j=1}^{m_{i}} \gamma_{i, j}(x)+\sum_{i=2}^{s}\left(-m_{1}+m_{i}+1\right) \gamma_{i}^{\prime}(x)+\sum_{i=2}^{s}\left(-m_{1}+m_{i}+1\right) \beta_{i} \\
& =C(x)+\sum_{i=2}^{s}\left(-m_{1}+m_{i}+1\right) \beta_{i} .
\end{aligned}
$$

Therefore, we obtain from (4.5) that $\mathrm{L}_{\mathcal{A}}(x)$ has the form given in (4.6).

4. Claim: $\mathcal{A}=\mathcal{A}(H)$.

Indeed, since $H$ is reduced (by Step 2) and $H=[\mathcal{A}]$, we have $\mathcal{A}(H) \subseteq \mathcal{A}$. Conversely, let $u \in \mathcal{A}$. We want to show that $\mathrm{L}_{\mathcal{A}}(u)=\{1\}$, which implies that $u \in \mathcal{A}(H)$.

To this end, assume first that $u=u_{k, l}$ for some $k \in \llbracket 1, s \rrbracket$ and $l \in \llbracket 1, m_{k} \rrbracket$. Then, $\gamma_{i}(u)=\gamma_{i}^{\prime}(u)=0$ for all $i \in \llbracket 1, s \rrbracket$, because $m_{i} \geq 2$. Thus, $C(u)=1$ and $k(u)=0$, and hence $\mathrm{L}_{\mathcal{A}}(u)=\{1\}$ by $(4.6)$.

Next, suppose that $u=u_{k, 0}$ for some $k \in \llbracket 2, s \rrbracket$. Then we have

$$
\gamma_{i}(u)= \begin{cases}1 & \text { if } i=1 \\ -1 & \text { if } i=k \\ 0 & \text { if } i \neq 1, k\end{cases}
$$

Thus, we find that $\gamma_{k}^{\prime}(u)=1, \gamma_{i}^{\prime}(u)=0$ if $i \neq k$, and $k(u)=0$. Consequently, we have by (4.6) that

$$
\mathrm{L}_{\mathcal{A}}(u)=\{C(u)\}=\left\{m_{1}-m_{k}+\left(-m_{1}+m_{k}+1\right)\right\}=\{1\} .
$$

5. Minima and maxima of sets of lengths. Let $x \in H$. Since $\mathcal{A}=\mathcal{A}(H)$ by Step 4 , we have

$$
\mathrm{L}_{H}(x)=\mathrm{L}_{\mathcal{A}}(x)=C(x)+\left\{\sum_{i=2}^{s}\left(-m_{1}+m_{i}+1\right) \beta_{i}: \beta_{2}, \ldots, \beta_{s} \in \mathbb{N}_{0} \text { and } \sum_{i=2}^{s} \beta_{i} \leq k(x)\right\},
$$

and we assert that

$$
\min \mathrm{L}_{H}(x)=C(x), \quad \max \mathrm{L}_{H}(x)=C(x)+k(x)\left(-m_{1}+m_{s}+1\right), \quad \text { and } \quad C(x) \geq k(x) m_{1} .
$$


Indeed, the first equality follows immediately from (4.7). As for the second, it is clear that

$$
C(x)+k(x)\left(-m_{1}+m_{s}+1\right) \in \mathrm{L}_{H}(x),
$$

because $0+\ldots+0+k(x) \leq k(x)$. Conversely, if $\beta_{2}, \ldots, \beta_{s} \in \mathbb{N}_{0}$ and $\sum_{i=2}^{s} \beta_{i} \leq k(x)$, then

$$
C(x)+\sum_{i=2}^{s}\left(-m_{1}+m_{i}+1\right) \beta_{i} \leq C(x)+\left(-m_{1}+m_{s}+1\right) \sum_{i=2}^{s} \beta_{i} \leq C(x)+k(x)\left(-m_{1}+m_{s}+1\right),
$$

where we have used that $m_{1} \leq \ldots \leq m_{s}$ (by construction). Therefore, $\max L_{H}(x)$ is as stated.

Finally (recall that $\gamma_{i}^{\prime}(x)=\max \left\{0,-\gamma_{i}(x)\right\}$, and hence $\gamma_{i}(x) \geq-\gamma_{i}^{\prime}(x)$ ), we have

$$
\begin{aligned}
C(x) & =\sum_{i=1}^{s} \sum_{j=1}^{m_{i}} \gamma_{i, j}(x)+\sum_{i=2}^{s} \gamma_{i}^{\prime}(x)\left(-m_{1}+m_{i}+1\right) \geq \sum_{i=1}^{s} m_{i} \gamma_{i}(x)+\sum_{i=2}^{s} \gamma_{i}^{\prime}(x)\left(-m_{1}+m_{i}+1\right) \\
& \geq m_{1} \gamma_{1}(x)-\sum_{i=2}^{s} \gamma_{i}^{\prime}(x) m_{i}+\sum_{i=2}^{s} \gamma_{i}^{\prime}(x)\left(-m_{1}+m_{i}+1\right)=m_{1} \gamma_{1}(x)-\left(m_{1}-1\right) \sum_{i=2}^{s} \gamma_{i}^{\prime}(x) \\
& \geq m_{1}\left(\gamma_{1}(x)-\sum_{i=2}^{s} \gamma_{i}^{\prime}(x)\right)=m_{1} k(x) .
\end{aligned}
$$

6. Claim: $L \in \mathscr{L}(H)$ and $\mathscr{U}_{\min L}(H)=L$.

Note that $\min L=m_{1}$, and set $x=u_{1,1} \cdot \ldots \cdot u_{1, m_{1}}$. Then $\gamma_{1}(x)=1, \gamma_{i}^{\prime}(x)=0$ for each $i \in \llbracket 2, s \rrbracket$, $C(x)=m_{1}$, and $k(x)=1$. So we have by (4.7) that

$$
\mathrm{L}_{H}(x)=m_{1}+\left\{0,-m_{1}+m_{2}+1, \ldots,-m_{1}+m_{s}+1\right\}=L .
$$

Since $m_{1} \in \mathrm{L}_{H}(x)=L$, we obtain $L \subseteq \mathscr{U}_{m_{1}}(H)$.

Conversely, let $x \in H$ such that $m_{1} \in \mathrm{L}_{H}(x)$. We need to show that $\mathrm{L}_{H}(x) \subseteq L$. Since $m_{1} \in \mathrm{L}_{H}(x)$, we get from (4.8) and (4.7) that

$$
k(x) m_{1} \leq C(x)=\min \mathrm{L}_{H}(x) \leq m_{1} .
$$

It follows that $k(x)=0$ or $k(x)=1$, and this concludes the proof of the claim, as we have by (4.7) and the above that $\mathrm{L}_{H}(x)=\{C(x)\}=\left\{m_{1}\right\} \subseteq L$ if $k(x)=0$, and $L_{H}(x)=L$ otherwise.

7. Claim: $\max \Delta(H)=\max \Delta(L)$.

Since $L \in \mathscr{L}(H)$ by the previous step, it is enough to prove that $\max \Delta(H) \leq \max \Delta(L)$. Let $x \in H$ such that $\Delta\left(\mathrm{L}_{H}(x)\right)$ is non-empty, and let $l<l^{\prime}$ be consecutive elements of $\mathrm{L}_{H}(x)$. We have to show that $l^{\prime}-l \leq \max \Delta(L)$. To this end, recall (4.7) and pick $\beta_{2}, \ldots, \beta_{s} \in \mathbb{N}_{0}$ such that $\sum_{i=2}^{s} \beta_{i} \leq k(x)$ and $l=C(x)+\sum_{i=2}^{s}\left(-m_{1}+m_{i}+1\right) \beta_{i}$. We distinguish two cases.

CASE 1: $\beta_{i_{0}}>0$ for some $i_{0} \in \llbracket 2, s-1 \rrbracket$.

For every $i \in \llbracket 2, s \rrbracket$, we define $\beta^{\prime}=\left(\beta_{2}^{\prime}, \ldots, \beta_{s}^{\prime}\right)$ by

$$
\beta_{i}^{\prime}= \begin{cases}\beta_{i} & \text { if } i \neq i_{0}, i_{0}+1 \\ \beta_{i_{0}}-1 & \text { if } i=i_{0} \\ \beta_{i_{0}+1}+1 & \text { if } i=i_{0}+1\end{cases}
$$


Then $\beta^{\prime} \in \mathbb{N}_{0}^{s-1}$ and $\sum_{i=2}^{s} \beta_{i}^{\prime}=\sum_{i=2}^{s} \beta_{i} \leq k(x)$, so we obtain from (4.7) that

$$
\begin{aligned}
\mathrm{L}_{H}(x) \ni l^{\prime \prime} & :=C(x)+\sum_{i=2}^{s}\left(-m_{1}+m_{i}+1\right) \beta_{i}^{\prime} \\
& =C(x)+\sum_{i=2}^{s}\left(-m_{1}+m_{i}+1\right) \beta_{i}-\left(-m_{1}+m_{i_{0}}+1\right)+\left(-m_{1}+m_{i_{0}+1}+1\right) \\
& =l+\left(m_{i_{0}+1}+1\right)-\left(m_{i_{0}}+1\right) .
\end{aligned}
$$

Using that $m_{i}<m_{i+1}$ for every $i \in \llbracket 2, s-1 \rrbracket$, it follows that $l<l^{\prime} \leq l^{\prime \prime}$, and therefore

$$
l^{\prime}-l \leq l^{\prime \prime}-l=\left(m_{i_{0}+1}+1\right)-\left(m_{i_{0}}+1\right) \leq \max \Delta(L) .
$$

CASE 2: $\beta_{i}=0$ for each $i \in \llbracket 2, s-1 \rrbracket$.

Then $l=C(x)+\left(-m_{1}+m_{s}+1\right) \beta_{s}$, and we have $\beta_{s}<k(x)$, since we get from (4.8) that

$$
l<\max \mathrm{L}_{H}(x)=C(x)+k(x)\left(-m_{1}+m_{s}+1\right) .
$$

It follows that $1+\beta_{s} \leq k(x)$, and then we infer from (4.7) that

$$
\mathrm{L}_{H}(x) \ni l^{\prime \prime}:=C(x)+\left(-m_{1}+m_{2}+1\right)+\left(-m_{1}+m_{s}+1\right) \beta_{s}=l+\left(-m_{1}+m_{2}+1\right)>l .
$$

So $l<l^{\prime} \leq l^{\prime \prime}$ and $l^{\prime}-l \leq l^{\prime \prime}-l=\left(m_{2}+1\right)-m_{1} \leq \max \Delta(L)$.

8. Claim: $\rho_{\ell \min L+\nu}(H)=\ell \max L+\nu$ for each $\nu \in \llbracket 0, \min L-1 \rrbracket$ and all $\ell \in \mathbb{N}_{0}$.

Clearly, we have $\min L=m_{1}$ and $\max L=m_{s}+1$. Let $\ell \in \mathbb{N}_{0}$ and $\nu \in \llbracket 0, m_{1}-1 \rrbracket$. From $u_{s, 0}=$ $\left(u_{1,1} \cdot \ldots \cdot u_{1, m_{1}}\right)\left(u_{s, 1} \cdot \ldots \cdot u_{s, m_{s}}\right)^{-1}$ we obtain

$$
\left(u_{1,1} \cdot \ldots \cdot u_{1, m_{1}}\right)^{\ell}\left(u_{1,1} \cdot \ldots \cdot u_{1, \nu}\right)=\left(u_{s, 0} u_{s, 1} \cdot \ldots \cdot u_{s, m_{s}}\right)^{\ell}\left(u_{1,1} \cdot \ldots \cdot u_{1, \nu}\right) .
$$

Since $\mathcal{A}(H)=\mathcal{A}$ by Step 4, we thus find that $\rho_{\ell m_{1}+\nu}(H) \geq \ell\left(m_{s}+1\right)+\nu$.

Conversely, let $x \in H$ be such that $\ell m_{1}+\nu \in \mathrm{L}_{H}(x)$. It follows from Step 5 that

$$
\ell m_{1}+\nu \geq \min \mathrm{L}_{H}(x)=C(x) \geq k(x) m_{1},
$$

which implies $k(x) \leq \ell$. Therefore, we have by (4.8) that

$$
\max \mathrm{L}_{H}(x)=C(x)+k(x)\left(-m_{1}+m_{s}+1\right) \leq \ell m_{1}+\nu+\ell\left(-m_{1}+m_{s}+1\right)=\ell\left(m_{s}+1\right)+\nu,
$$

which, in turn, implies $\rho_{\ell m_{1}+\nu}(H) \leq \ell\left(m_{s}+1\right)+\nu$.

Theorem 4.2. Let $d \in \mathbb{N}_{\geq 2}$ and let $\left(m_{k}\right)_{k \geq 0}$ be a sequence of non-negative integers such that $m_{0}=0$, $m_{1}=1$, and

$$
\sum_{i=1}^{k-2}\left(\frac{k}{i} m_{i}+(i-1)\right) \leq m_{k-1}-(k-1) \quad \text { for each } \quad k \geq 3 .
$$

Moreover, let $\left(U_{k}\right)_{k \geq 1}$ be a family of finite subsets of the positive integers with $U_{1}=\varnothing$ such that, for all $k \geq 2, U_{k}$ is non-empty, $m_{k-1}+1<\min U_{k} \leq m_{k-1}+d$, $\max U_{k}=m_{k}$, and $\max \Delta\left(U_{k}\right) \leq d$. Then there exists a locally tame Krull monoid $H$ with finite set of distances $\Delta(H) \subseteq \llbracket 1, d \rrbracket$ and with

$$
\mathscr{U}_{k}(H) \cap \mathbb{N}_{\geq k}=\llbracket k, m_{k-1}+1 \rrbracket \uplus U_{k} \quad \text { for each } \quad k \in \mathbb{N} .
$$


In particular, if $\min U_{k}+1 \notin U_{k}$ for all $k \geq 2$, then there exists no $M \in \mathbb{N}$ such that $\mathscr{U}_{k}(H)$ is an $\mathrm{AAP}$ with difference 1 and bound $M$ for all sufficiently large $k$, and hence $\mathscr{L}(H)$ does not satisfy the Structure Theorem for Unions.

Comment. Of course, we can combine the present result, showing the existence of a Krull monoid $H$ with prescribed properties for $\mathscr{L}(H)$, with Claborn's Realization Theorem for class groups ([23, Theorem 3.7.8]) or with one of the realization theorems for monoids of modules (mentioned in Subsection 3.3). Then we obtain a Dedekind domain $R$, respectively a monoid of modules $\mathcal{V}(\mathcal{C})$, such that $\mathscr{L}(R)$, respectively $\mathscr{L}(\mathcal{V}(\mathcal{C}))$, has the prescribed properties.

Proof. The "In particular" part is a simple consequence of the main statement.

As for the rest, let $d,\left(m_{k}\right)_{k \geq 0}$, and $\left(U_{k}\right)_{k \geq 1}$ be given with the above properties. For each $k \in \mathbb{N}$ we set

$$
L_{k}=\llbracket k, m_{k-1}+1 \rrbracket \uplus U_{k},
$$

and we note that $L_{1}=\{1\}$. In addition, we set $H_{1}=\left(\mathbb{N}_{0},+\right)$, and for every $k \geq 2$ we choose a finitely generated reduced Krull monoid $H_{k}$ with $L_{k} \in \mathscr{L}\left(H_{k}\right)$ that satisfies all the conditions specified by points (i)-(iii) of Proposition 4.1. We claim that the coproduct

$$
H=\coprod_{k \geq 1} H_{k}
$$

has all the required properties. Because finitely generated monoids are locally tame ([23, Theorem 3.1.4]) and the coproduct of locally tame Krull monoids is a locally tame Krull monoid ([23, Proposition 1.6.8]), the monoid $H$ is a locally tame Krull monoid. Furthermore, $\operatorname{since} \sup \Delta(H)=\sup \left\{\sup \Delta\left(H_{k}\right): k \in \mathbb{N}\right\}$ by [23, Proposition 1.4.5], it follows that $\Delta(H) \subseteq \llbracket 1, d \rrbracket$. So we are left with the statement on the unions $\mathscr{U}_{k}(H)$. For this, we will prove the following four assertions, the last of which implies the theorem.

(A1) For all $k \in \mathbb{N}$, we have $\mathscr{U}_{k}\left(H_{k}\right)=L_{k}$ and $\mathscr{U}_{\nu}\left(H_{k}\right)=\{\nu\}$ for $\nu \in \llbracket 1, k-1 \rrbracket$.

(A2) $\rho_{k}\left(H_{1} \times \ldots \times H_{k-1}\right)=1+m_{k-1}$ for each $k \in \mathbb{N}_{\geq 2}$.

(A3) $\mathscr{U}_{k}\left(H_{1} \times \ldots \times H_{k}\right) \cap \mathbb{N}_{\geq k}=L_{k}$ for every $k \in \mathbb{N}$.

(A4) $\mathscr{U}_{k}(H) \cap \mathbb{N}_{\geq k}=L_{k}$ for all $k \in \mathbb{N}$.

To start with, we recall that, for every atomic monoid $S$ with $S \neq S^{\times}$, we have $\mathscr{U}_{1}(S)=\{1\}, \mathscr{U}_{0}(S)=$ $\{0\}$, and $\rho_{0}(S)=0$. Note that, by construction, the monoids $H_{k}$ are reduced, and hence $H_{k} \neq H_{k}^{\times}=\{1\}$, for all $k \in \mathbb{N}$.

Proof of (A1). This is straightforward from Conditions (ii) and (iii) of Proposition 4.1 (and the fact that, by construction, $\left.\min L_{k}=k\right)$.

Proof of (A2). The assertion holds for $k=2$, because $\rho_{2}\left(H_{1}\right)=2$ and $m_{1}=1$. So, let $k \geq 3$ and set $S=H_{1} \times \ldots \times H_{k-2}$. We want to show that

$$
\rho_{k}\left(S \times H_{k-1}\right)=\max \left\{\rho_{\nu}(S)+\rho_{k-\nu}\left(H_{k-1}\right): \nu \in \llbracket 0, k \rrbracket\right\}=1+m_{k-1} .
$$

To begin, we have by (A1) and Condition (iii) of Proposition 4.1 that

$$
\rho_{0}(S)+\rho_{k}\left(H_{k-1}\right)=1+m_{k-1}=\rho_{1}(S)+\rho_{k-1}\left(H_{k-1}\right)
$$


and $\rho_{k-\nu}\left(H_{k-1}\right)=k-\nu$ for every $\nu \in \llbracket 2, k \rrbracket$. Consequently, it is enough to prove that

$$
\rho_{\nu}(S) \leq m_{k-1}-(k-1)+\nu \quad \text { for each } \quad \nu \in \llbracket 2, k \rrbracket .
$$

To this end, let $i \in \llbracket 1, k-2 \rrbracket$ and write $k=\ell i+\nu$, where $\ell \in \mathbb{N}_{0}$ and $\nu \in \llbracket 0, i-1 \rrbracket$. Then, appealing again to Condition (iii) of Proposition 4.1, we find that

$$
\rho_{k}\left(H_{i}\right)=\rho_{\ell i+\nu}\left(H_{i}\right)=\ell m_{i}+\nu \leq \frac{k}{i} m_{i}+(i-1) .
$$

For each $\nu \in \llbracket 2, k \rrbracket$, we thus obtain that

$$
\rho_{\nu}(S) \leq \rho_{k}(S) \leq \sum_{i=1}^{k-2} \rho_{k}\left(H_{i}\right) \leq \sum_{i=1}^{k-2}\left(\frac{k}{i} m_{i}+(i-1)\right) \leq m_{k-1}-(k-1) .
$$

Proof of (A3). The statement holds for $k=1$. So, assume $k \geq 2$ and set $S=H_{1} \times \ldots \times H_{k-1}$. Then

$$
\begin{aligned}
\mathscr{U}_{k}\left(S \times H_{k}\right) \cap \mathbb{N}_{\geq k} & =\bigcup_{\nu=0}^{k}\left(\mathscr{U}_{\nu}(S)+\mathscr{U}_{k-\nu}\left(H_{k}\right)\right) \cap \mathbb{N}_{\geq k} \\
& =\left(\mathscr{U}_{k}\left(H_{k}\right) \cap \mathbb{N}_{\geq k}\right) \cup\left(\bigcup_{\nu=1}^{k}\left(\mathscr{U}_{\nu}(S)+\mathscr{U}_{k-\nu}\left(H_{k}\right)\right) \cap \mathbb{N}_{\geq k}\right) \\
& =\left(\mathscr{U}_{k}\left(H_{k}\right) \cap \mathbb{N}_{\geq k}\right) \cup\left(\bigcup_{\nu=1}^{k}\left((k-\nu)+\mathscr{U}_{\nu}(S)\right) \cap \mathbb{N}_{\geq k}\right) .
\end{aligned}
$$

Since for each $\nu \in \llbracket 1, k \rrbracket$ we have $(k-\nu)+\mathscr{U}_{\nu}(S) \subseteq \mathscr{U}_{k}(S)$, we see that

$$
L_{k} \subseteq \mathscr{U}_{k}\left(S \times H_{k}\right) \cap \mathbb{N}_{\geq k} \subseteq\left(\mathscr{U}_{k}\left(H_{k}\right) \cap \mathbb{N}_{\geq k}\right) \cup\left(\mathscr{U}_{k}(S) \cap \mathbb{N}_{\geq k}\right)=L_{k} \cup\left(\mathscr{U}_{k}(S) \cap \mathbb{N}_{\geq k}\right)=L_{k},
$$

where the last equality follows from (A2).

Proof of (A4). The statement is clear for $k=1$. So, suppose that $k \geq 2$, and set $S_{1}=H_{1} \times \ldots \times H_{k}$ and $S_{2}=\coprod_{\nu \geq k+1} H_{\nu}$, whence $H=S_{1} \times S_{2}$. If $\nu \in \llbracket 0, k \rrbracket$, then (A1) implies $\mathscr{U}_{\nu}\left(S_{2}\right)=\{\nu\}$. Therefore,

$$
\begin{aligned}
\mathscr{U}_{k}\left(S_{1} \times S_{2}\right) \cap \mathbb{N}_{\geq k} & =\bigcup_{\nu=0}^{k}\left(\mathscr{U}_{k-\nu}\left(S_{1}\right)+\mathscr{U}_{\nu}\left(S_{2}\right)\right) \cap \mathbb{N}_{\geq k} \\
& =\bigcup_{\nu=0}^{k}\left(\nu+\mathscr{U}_{k-\nu}\left(S_{1}\right)\right) \cap \mathbb{N}_{\geq k} \\
& =\left(\mathscr{U}_{k}\left(S_{1}\right) \cap \mathbb{N}_{\geq k}\right) \cup\left(\bigcup_{\nu=1}^{k}\left(\nu+\mathscr{U}_{k-\nu}\left(S_{1}\right)\right) \cap \mathbb{N}_{\geq k}\right),
\end{aligned}
$$

which, together with Lemma 2.4(2), shows that, for every $\nu \in \llbracket 1, k \rrbracket$,

$$
\nu+\mathscr{U}_{k-\nu}\left(S_{1}\right) \subseteq \mathscr{U}_{\nu}\left(S_{1}\right)+\mathscr{U}_{k-\nu}\left(S_{1}\right) \subseteq \mathscr{U}_{k}\left(S_{1}\right) .
$$

So, putting it all together, we get from (A3) that

$$
\mathscr{U}_{k}\left(S_{1} \times S_{2}\right) \cap \mathbb{N}_{\geq k}=\mathscr{U}_{k}\left(S_{1}\right) \cap \mathbb{N}_{\geq k}=L_{k} .
$$

This completes the proof of the theorem. 


\section{REFERENCES}

[1] D.D. Anderson and J.L. Mott, Cohen-Kaplansky Domains: Integral Domains with a Finite Number of Irreducible Elements, J. Algebra 148 (1992), no. 1, 17 - 41.

[2] N.R. Baeth and A. Geroldinger, Monoids of modules and arithmetic of direct-sum decompositions, Pacific J. Math. 271 (2014), no. 2, $257-319$.

[3] N.R. Baeth and R. Wiegand, Factorization Theory and Decomposition of Modules, Amer. Math. Monthly 120 (2013), no. $1,3-34$.

[4] P. Baginski, S.T. Chapman, N. Hine, and J. Paixão, On the asymptotic behavior of unions of sets of lengths in atomic monoids, Involve 1 (2008), no. 1, $101-110$.

[5] V. Blanco, P. A. García-Sánchez, and A. Geroldinger, Semigroup-theoretical characterizations of arithmetical invariants with applications to numerical monoids and Krull monoids, Illinois J. Math. 55 (2011), no. 4, 1385 - 1414.

[6] S. Breaz, G. Călugăreanu, and P. Schultz, Modules with Dedekind finite endomorphism rings, Mathematica 53(76) (2011), no. 1, $15-28$.

[7] S.T. Chapman, J. Daigle, R. Hoyer, and N. Kaplan, Delta Sets of Numerical Monoids Using Nonminimal Sets of Generators, Commun. Algebra 38 (2010), no. 7, $2622-2634$.

[8] S.T. Chapman and W.W. Smith, On the Lengths of Factorizations of Elements in an Algebraic Number Ring, J. Number Theory 43 (1993), no. 1, $24-30$.

[9] _ Generalized Sets of Lengths, J. Algebra 200 (1998), no. 2, $449-471$.

[10] L. Diracca, On a generalization of the exchange property to modules with semilocal endomorphism rings, J. Algebra 313 (2007), no. 2, $972-987$.

[11] A. Facchini, Direct sum decomposition of modules, semilocal endomorphism rings, and Krull monoids, J. Algebra 256 (2002), no. 1, $280-307$.

[12] — Geometric regularity of direct-sum decompositions in some classes of modules, J. Math. Sci. (N.Y.) 139 (2006), no. $4,6814-6822$.

[13] _ Krull Monoids and Their Application in Module Theory, Algebras, Rings and their Representations (A. Facchini, K. Fuller, C. M. Ringel, and C. Santa-Clara, eds.), World Scientific, 2006, pp. $53-71$.

[14] _ Direct-sum decompositions of modules with semilocal endomorphism rings, Bull. Math. Sci. 2 (2012), no. 2, $225-279$.

[15] A. Facchini and R. Wiegand, Direct-sum decomposition of modules with semilocal endomorphism rings, J. Algebra 274 (2004), no. 2, $689-707$.

[16] Y. Fan and S. Tringali, Power monoids: A bridge between Factorization Theory and Arithmetic Combinatorics, preprint (arXiv:1701.09152).

[17] Y. Fan and Q. Zhong, Products of $k$ atoms in Krull monoids, Monatsh. Math. 181 (2016), no. 4, 779 - 795.

[18] M. Freeze and A. Geroldinger, Unions of sets of lengths, Funct. Approx. Comment. Math. 39 (2008), no. 1,149 - 162.

[19] W. Gao and A. Geroldinger, On products of $k$ atoms, Monatsh. Math. 156 (2009), no. 2, $141-157$.

[20] A. Geroldinger, Sets of lengths, Amer. Math. Monthly 123 (2016), no. 10, 960 - 988.

[21] _ Additive Group Theory and Non-unique Factorizations, Combinatorial Number Theory and Additive Group Theory (A. Geroldinger and I. Ruzsa, eds.), Adv. Courses Math. CRM Barcelona, Birkhäuser, Basel, 2009 , pp. 1 - 86.

[22] A. Geroldinger, D.J. Grynkiewicz, and P. Yuan, On products of $k$ atoms II, Mosc. J. Comb. Number Theory 5 (2015), no. $3,73-129$.

[23] A. Geroldinger and F. Halter-Koch, Non-Unique Factorizations. Algebraic, Combinatorial and Analytic Theory, Pure Appl. Math., vol. 278, Chapman \& Hall/CRC, 2006.

[24] A. Geroldinger and W. Hassler, Local tameness of v-noetherian monoids, J. Pure Appl. Algebra 212 (2008), no. 6, 1509 -1524 .

[25] A. Geroldinger and F. Kainrath, On the arithmetic of tame monoids with applications to Krull monoids and Mori domains, J. Pure Appl. Algebra 214 (2010), no. 12, 2199 - 2218.

[26] A. Geroldinger, F. Kainrath, and A. Reinhart, Arithmetic of seminormal weakly Krull monoids and domains, J. Algebra 444 (2015), $201-245$.

[27] A. Geroldinger and G. Lettl, Factorization problems in semigroups, Semigroup Forum 40 (1990), no. 1, 23 - 38. 
[28] A. Geroldinger, S. Ramacher, and A. Reinhart, On v-Marot Mori rings and C-rings, J. Korean Math. Soc. 52 (2015), no. $1,1-21$.

[29] A. Geroldinger and W.A. Schmid, The system of sets of lengths in Krull monoids under set addition, Rev. Mat. Iberoam. 32 (2016), no. 2, $571-588$.

[30] K.R. Goodearl, Von Neumann Regular Rings, Monogr. Stud. Math., vol. 4, Pitman, 1979.

[31] D.J. Grynkiewicz, Structural Additive Theory, Developments in Mathematics, vol. 30, Springer, 2013.

[32] F. Halter-Koch, Ideal Systems. An Introduction to Multiplicative Ideal Theory, Marcel Dekker, 1998.

[33] _ Quadratic Irrationals. An Introduction to Classical Number Theory, Pure Appl. Math., vol. 306, Chapman \& Hall/CRC, 2013.

[34] M. Hazewinkel, N. Gubareni, and V.V. Kirichenko, Algebras, rings and modules. Vol. 1, Kluwer Academic Publishers, 2004.

[35] F. Kainrath, Arithmetic of Mori Domains and Monoids: The Global Case, Multiplicative Ideal Theory and Factorization Theory (S. Chapman, M. Fontana, A. Geroldinger, and B. Olberding, eds.), Springer Proc. Math. Stat., vol. 170, Springer, 2016, pp. $183-218$.

[36] G. Pólya and G. Szegő, Problems and Theorems in Analysis I: Series. Integral Calculus. Theory of Functions, Springer, 1998.

[37] A. Reinhart, On integral domains that are C-monoids, Houston J. Math. 39 (2013), no. 4, 1095 - 1116.

[38] J.C. Rosales, P.A. García-Sánchez, and J.I. García-García, Atomic Commutative Monoids and Their Elasticity, Semigroup Forum 68 (2004), no. 1, $64-86$.

[39] D. Smertnig, Sets of lengths in maximal orders in central simple algebras, J. Algebra 390 (2013), 1- 43.

[40] R. Wiegand and S. Wiegand, Semigroups of Modules: a Survey, Rings, Modules and Representations (N.V. Dung, F. Guerriero, L. Hammoudi, and P. Kanwar, eds.), Contemp. Math., vol. 480, Amer. Math. Soc., 2009, pp. 335 - 349.

Mathematical College, China University of Geosciences | Haidian District, Beijing, China

E-mail address: fys@cugb.edu.cn

$U R L:$ http://yushuang-fan.weebly.com/

Institute for Mathematics and Scientific Computing, University of Graz | Heinrichstr. 36, 8010 Graz, Austria

E-mail address: alfred.geroldinger@uni-graz.at

$U R L$ : http://imsc.uni-graz.at/geroldinger

Institute for Mathematics and Scientific Computing, University of Graz | Heinrichstr. 36, 8010 Graz, Austria

E-mail address: florian.kainrath@uni-graz.at

Institute for Mathematics and Scientific Computing, University of Graz | Heinrichstr. 36, 8010 Graz, Austria

E-mail address: salvatore.tringali@uni-graz.at

$U R L$ : http://imsc.uni-graz.at/tringali/ 